\title{
Quantum mechanics on spaces of nonconstant curvature: the oscillator problem and superintegrability
}

\author{
Ángel Ballesteros ${ }^{a}$, Alberto Enciso ${ }^{b}$, Francisco J. Herranz ${ }^{a}$, \\ Orlando Ragnisco ${ }^{c}$ and Danilo Riglioni ${ }^{c}$ \\ ${ }^{a}$ Departamento de Física, Universidad de Burgos, 09001 Burgos, Spain \\ E-mail: angelb@ubu.es fjherranz@ubu.es \\ ${ }^{b}$ Departamento de Física Teórica II, Universidad Complutense, 28040 Madrid, Spain \\ E-mail: aenciso@fis.ucm.es \\ ${ }^{c}$ Dipartimento di Fisica, Università di Roma Tre and Istituto Nazionale di Fisica Nucleare \\ sezione di Roma Tre, Via Vasca Navale 84, 00146 Roma, Italy \\ E-mail: ragnisco@fis.uniroma3.it riglioni@fis.uniroma3.it
}

\begin{abstract}
The full spectrum and eigenfunctions of the quantum version of a nonlinear oscillator defined on an $N$-dimensional space with nonconstant curvature are rigorously found. Since the underlying curved space generates a position-dependent kinetic energy, three different quantization prescriptions are worked out by imposing that the maximal superintegrability of the system has to be preserved after quantization. The relationships among these three Schrödinger problems are described in detail through appropriate similarity transformations. These three approaches are used to illustrate different features of the quantization problem on $N$-dimensional curved spaces or, alternatively, of position-dependent mass quantum Hamiltonians. This quantum oscillator is, to the best of our knowledge, the first example of a maximally superintegrable quantum system on an $N$-dimensional space with nonconstant curvature.
\end{abstract}

PACS: 03.65.-w 02.30.Ik 05.45.-a

KEYWORDS: nonlinear, oscillator, superintegrability, deformation, hyperbolic, curvature, position-dependent mass 


\section{Introduction}

This paper is devoted to the study of the quantum mechanical version of $N$-dimensional $(N D)$ classical Hamiltonian systems of the type

$$
\mathcal{H}(\mathbf{q}, \mathbf{p})=\mathcal{T}(\mathbf{q}, \mathbf{p})+\mathcal{U}(\mathbf{q})=\frac{\mathbf{p}^{2}}{2 \mathcal{M}(\mathbf{q})}+\mathcal{U}(\mathbf{q})
$$

where $\mathbf{q}, \mathbf{p} \in \mathbb{R}^{N}$ are conjugate coordinates and momenta with canonical Poisson bracket $\left\{q_{i}, p_{j}\right\}=\delta_{i j}$. The physical interpretation of these systems is two-fold. On one hand, as the Hamiltonian describing the motion of a particle on the ND curved space defined by the (conformally flat) metric $\mathrm{d} s^{2}=\mathcal{M}(\mathbf{q}) \mathrm{d} \mathbf{q}^{2}$ and under the action of the potential $\mathcal{U}(\mathbf{q})$. On the other, as position-dependent mass systems on the ND Euclidean space.

Evidently, the crucial point for the definition of the corresponding Schrödinger problem is the consistent (under certain given criteria) definition of the quantum kinetic energy term, $\mathcal{T}(\mathbf{q}, \mathbf{p}) \rightarrow \hat{\mathcal{T}}(\hat{\mathbf{q}}, \hat{\mathbf{p}})$, since an obvious ordering ambiguity appears when the position and momenta operators are considered. In this paper we shall deal with a specific example: the motion of a particle on the so-called ND Darboux III space [1, 2] given by $\mathcal{M}(\mathbf{q})=1+\lambda \mathbf{q}^{2}$ (with $\lambda>0$ ), and whose potential will be the intrinsic oscillator on such space defined by

$$
\mathcal{U}(\mathbf{q})=\frac{\omega^{2} \mathbf{q}^{2}}{2\left(1+\lambda \mathbf{q}^{2}\right)}
$$

This choice is motivated by the fact that this system is the only known example of a maximally superintegrable classical Hamiltonian on an ND space with nonconstant curvature [3, and it can be interpreted as a $\lambda$-deformation of the flat isotropic oscillator, which is recovered in the $\lambda \rightarrow 0$ limit. In fact, it is well-known that the quantum superintegrability of the $N \mathrm{D}$ flat isotropic oscillator is useful in order to obtain its exact solution by making use of the superabundance of quantum integrals of the motion [4. Therefore, it seems natural to quantize the Darboux III oscillator in such a way that the maximal superintegrability of the system will be manifestly preserved under quantization; moreover, presumably in this way this new ND nonlinear oscillator could be fully solved by mimicking the standard procedure for the Euclidean oscillator.

In the sequel we will show that this is indeed the case. In fact, the superintegrability constraint will be useful in order to analyse in detail several possible quantization prescriptions amenable for ND spaces with nonconstant curvature, namely:

- The so-called [5] direct "Schrödinger" quantization

$$
\hat{\mathcal{H}}=\frac{1}{2\left(1+\lambda \hat{\mathbf{q}}^{2}\right)} \hat{\mathbf{p}}^{2}+\frac{\omega^{2} \hat{\mathbf{q}}^{2}}{2\left(1+\lambda \hat{\mathbf{q}}^{2}\right)}=\frac{1}{2\left(1+\lambda \mathbf{q}^{2}\right)}\left(-\hbar^{2} \Delta+\omega^{2} \mathbf{q}^{2}\right),
$$

which was the one used in [6] for this system, since it preserves the maximal superintegrability in a straightforward way due to the immediate quantum transcription of the $(2 N-1)$ classical integrals of the motion. This property leads to a maximal degeneracy of the spectrum, which is exactly the same as in the quantum ND flat oscillator. 
- The "Laplace-Beltrami" (LB) quantization, which makes use of the usual LB operator on curved spaces:

$$
\hat{\mathcal{H}}_{\mathrm{LB}}=-\frac{\hbar^{2}}{2} \Delta_{\mathrm{LB}}+\frac{\omega^{2} \mathbf{q}^{2}}{2\left(1+\lambda \mathbf{q}^{2}\right)} \quad \text { where } \quad \Delta_{\mathrm{LB}}=\sum_{i, j=1}^{N} \frac{1}{\sqrt{g}} \partial_{i} \sqrt{g} g^{i j} \partial_{j} .
$$

However, we shall show that this LB Hamiltonian cannot be transformed into (1.1) through a similarity transformation unless we include an additional quantum potential which is proportional to the scalar curvature of the underlying space. This similarity transformation guarantees that the spectra of (1.2) and 1.1) coincide, and provides the explicit form of the full set of quantum integrals for $\mathcal{H}_{\mathrm{LB}}$. We stress that such a kind of quantum "geometric" potential is well-known in the literature (both in scalar field theories in General Relativity as well as in the context of the quantization problem on generic Riemannian manifolds [7, 8, 9]), and it is tantamount to replace the LB operator by the so-called "conformal Laplacian" [10]. We have to mention here that the connection between LB operators and scalar curvatures associated with two different, possibly conformally flat, Riemannian manifolds has been firstly pointed out in a pioneering paper by Paneitz in 1983 [11. However, the neat connection of the latter with quantum superintegrability properties is here stated for the first time and we think that this result opens the path for a novel algebraic approach to the subject.

- Finally, a "position-dependent mass" (PDM) quantization, which is essential in many condensed matter problems (see for instance [12, 13, 14, 15, 16, 17]). Here we perform it by considering the symmetric prescription proposed in [15], namely:

$$
\hat{\mathcal{H}}_{\mathrm{PDM}}(\hat{\mathbf{q}}, \hat{\mathbf{p}})=\frac{1}{2} \hat{\mathbf{p}} \cdot \frac{1}{\left(1+\lambda \hat{\mathbf{q}}^{2}\right)} \hat{\mathbf{p}}+\frac{\omega^{2} \hat{\mathbf{q}}^{2}}{2\left(1+\lambda \hat{\mathbf{q}}^{2}\right)}=-\frac{\hbar^{2}}{2} \nabla \cdot \frac{1}{\left(1+\lambda \mathbf{q}^{2}\right)} \nabla+\frac{\omega^{2} \mathbf{q}^{2}}{2\left(1+\lambda \mathbf{q}^{2}\right)} .
$$

In this case we again find that, in order to get a similarity transformation leading to (1.1), another additional quantum potential has to be added to (1.3). In this way the spectrum is preserved and the full set of quantum integrals of the motion is explicitly obtained.

We stress that although the three previous quantum Hamiltonians have different explicit expressions and, to some extent, interpretations, all of them are related through similarity transformations provided the additional quantum potential terms are considered. This, in turn, means that they share a common energy spectrum, but they have different wave functions. Moreover, one of the main objectives of this comparative analysis is to point out some generic (i.e. potential independent) features of the quantization problem on curved spaces, such as the relevance of the dimension $N$ of the underlying manifold (the $N=2$ case will be distinguished) as well as the particular properties of the nonconstant curvature cases.

The paper is organized as follows. In the next Section, the classical Darboux III oscillator is revisited in order to provide the necessary classical background on this system, including the explicit description of its maximal superintegrability in terms of a curved Fradkin tensor. Section 3 is devoted to review the geometry of the underlying space, emphasizing the role of its nonconstant curvature and introducing a classical radial effective potential that will be useful in the quantum context. In Section 4 the three superintegrable quantizations of 
the Darboux III oscillator are obtained, and the similarity transformations among them are fully described. Section 5 provides the three associated radial Schrödinger equations. The spectrum and eigenfunctions of the system are rigorously obtained in Section 6, thus completing the preliminary results given in [6]. Finally, Section 7 includes several remarks and open problems.

\section{The Darboux III oscillator}

The ND classical Hamiltonian system given by

$$
H(\mathbf{q}, \mathbf{p})=\frac{\mathbf{p}^{2}+\omega^{2} \mathbf{q}^{2}}{\kappa+\mathbf{q}^{2}}
$$

with real parameters $\kappa>0$ and $\omega \geq 0$, was proven in [3] to be maximally superintegrable (MS), since this Hamiltonian is endowed with the maximum possible number of $2 N-1$ functionally independent constants of motion. Hereafter we shall consider the equivalent Hamiltonian $\mathcal{H}$ defined by $\mathcal{H}=\kappa H / 2$ with real parameter $\lambda=1 / \kappa>0$ :

$$
\mathcal{H}(\mathbf{q}, \mathbf{p})=\mathcal{T}(\mathbf{q}, \mathbf{p})+\mathcal{U}(\mathbf{q})=\frac{\mathbf{p}^{2}}{2\left(1+\lambda \mathbf{q}^{2}\right)}+\frac{\omega^{2} \mathbf{q}^{2}}{2\left(1+\lambda \mathbf{q}^{2}\right)}
$$

The kinetic energy $\mathcal{T}(\mathbf{q}, \mathbf{p})$ can be interpreted as the one generating the geodesic motion of a particle with unit mass on a conformally flat space with metric and (nonconstant) scalar curvature given by

$$
\mathrm{d} s^{2}=\left(1+\lambda \mathbf{q}^{2}\right) \mathrm{d} \mathbf{q}^{2}, \quad R(\mathbf{q})=-\lambda \frac{(N-1)\left(2 N+3(N-2) \lambda \mathbf{q}^{2}\right)}{\left(1+\lambda \mathbf{q}^{2}\right)^{3}} .
$$

In fact, such a curved space is the ND spherically symmetric generalization of the Darboux surface of type III [18, 19, which was constructed in [1, 2]. On the other hand, the central potential $\mathcal{U}$ was proven in [2, 3] to be an "intrinsic" oscillator potential on that Darboux space.

Moreover, in spite of the very naive appearance of the classical Hamiltonian (2.2), which is nothing but the ND isotropic harmonic oscillator system divided by its oscillator potential (plus a rather relevant constant here scaled to 1), it is worth mentioning that this system can also be considered in three other different (but related) frameworks:

- For $N=3, \mathcal{H}$ arises as a particular case of the so call multifold (or $\nu$-fold) Kepler 3D Hamiltonians constructed in [5, 20] as generalizations of the MIC-Kepler and TaubNUT systems [21, 22, 23, 24, 25, 26, 27, 28, 29]. In the notation of [20], $\mathcal{H}$ can be recovered by setting $\nu=1 / 2, a=1$ and $b=\lambda$. Notice that from our approach, the proper Kepler-Coulomb potential on the Darboux space 2.3) would be [2]

$$
\mathcal{U}_{\mathrm{KC}}=\alpha \frac{\sqrt{1+\lambda \mathbf{q}^{2}}}{|\mathbf{q}|}, \quad|\mathbf{q}|=\sqrt{\mathbf{q}^{2}}, \quad \alpha \in \mathbb{R},
$$

which is related with the curved oscillator potential through $\mathcal{U} \propto \mathcal{U}_{\mathrm{KC}}^{-2}$. 
- Again for $N=3$, the potential $\mathcal{U}$ can be obtained from the temporal part of the family of the so called Bertrand metrics [30, 31] on $(3+1)$ D Lorentzian spacetimes with nonconstant curvature. Such $(3+1)$ D free systems possess stable circular orbits and all of their bounded trajectories are periodic, so these are the natural generalization of the classical Bertrand's theorem 32 to spaces of nonconstant curvature. We recall that the MS property for of all 3D Bertrand Hamiltonians, which come from the $(3+1) \mathrm{D}$ Bertrand metrics, was recently proven in [33] (see also [34]).

- As we have already mentioned, $\mathcal{H}$ can alternatively be interpreted as describing a PDM system in which the conformal factor of the metric (2.3) is identified with the mass function, which in this case is parabolic: $\mathcal{M}(\mathbf{q})=1+\lambda \mathbf{q}^{2}$.

We point out that we have chosen to deal with 2.2 instead of (2.1) because in this way all the expressions that we shall present throughout the paper will have a smooth and well defined limit $\lambda \rightarrow 0$ that leads to the well-known results concerning the (flat) ND isotropic harmonic oscillator with frequency $\omega$. In particular, the limit $\lambda \rightarrow 0$ of $(2.2)$ and $(2.3)$ yields

$$
\mathcal{H}_{0}=\frac{1}{2} \mathbf{p}^{2}+\frac{1}{2} \omega^{2} \mathbf{q}^{2}, \quad \mathrm{~d} s^{2}=\mathrm{d} \mathbf{q}^{2}, \quad R=0 .
$$

\subsection{Maximal superintegrability from a curved Fradkin tensor}

The fact that $\mathcal{H}$ is a MS Hamiltonian can be explicitly demonstrated as follows [3, 35].

Theorem 1. (i) The Hamiltonian $\mathcal{H}$ (2.2) is endowed with the following constants of motion. - $(2 N-3)$ angular momentum integrals:

$$
C^{(m)}=\sum_{1 \leq i<j \leq m}\left(q_{i} p_{j}-q_{j} p_{i}\right)^{2}, \quad C_{(m)}=\sum_{N-m<i<j \leq N}\left(q_{i} p_{j}-q_{j} p_{i}\right)^{2},
$$

where $m=2, \ldots, N$ and $C^{(N)}=C_{(N)}$.

- $N^{2}$ integrals which form the ND curved Fradkin tensor:

$$
I_{i j}=p_{i} p_{j}-\left(2 \lambda \mathcal{H}(\mathbf{q}, \mathbf{p})-\omega^{2}\right) q_{i} q_{j},
$$

where $i, j=1, \ldots, N$ and such that $\mathcal{H}=\frac{1}{2} \sum_{i=1}^{N} I_{i i}$.

(ii) Each of the three sets $\left\{\mathcal{H}, C^{(m)}\right\},\left\{\mathcal{H}, C_{(m)}\right\}(m=2, \ldots, N)$ and $\left\{I_{i i}\right\}(i=1, \ldots, N)$ is formed by $N$ functionally independent functions in involution.

(iii) The set $\left\{\mathcal{H}, C^{(m)}, C_{(m)}, I_{i i}\right\}$ for $m=2, \ldots, N$ with a fixed index $i$ is constituted by $2 N-1$ functionally independent functions.

Notice that the first set of $2 N-3$ integrals 2.5 is the same for any central potential on any spherically symmetric space $[2$ since it is provided by an underlying $\mathfrak{s l}(2, \mathbb{R})$ coalgebra symmetry (also by an $\mathfrak{s o}(N)$-symmetry), while the second one (2.6) comes from the specific oscillator potential that we consider here. The latter, in fact, correspond to a curved analog of the Fradkin tensor of integrals of motion [4] for the isotropic harmonic oscillator. We also recall that the Hamiltonian (2.2) together with both sets of integrals of $(2.5)$ and $(2.6)$ can 
alternatively be obtained [35] from the free Euclidean motion by means of a Stäckel transform or coupling constant metamorphosis (see [36, 37, 38, 39] and references therein).

Thus, in general, the latter integrals (2.6) do not exist for a generic central potential so that, in principle, the MS property is not ensured at all. From this viewpoint the ND nonlinear oscillator Hamiltonian $\mathcal{H}(2.2)$ can be regarded as the "closest neighbour" of nonconstant curvature to the isotropic harmonic oscillator system (2.4) (with $\lambda=0$ ) as both share the same MS property. In fact, the real parameter $\lambda$ behaves as a "deformation" parameter governing the nonlinear behaviour of $\mathcal{H}$, and this parameter is deeply related to the variable curvature of the underlying Darboux space.

\subsection{Expressions in terms of hyperspherical coordinates in phase space}

The above results can also be expressed in terms of hyperspherical coordinates $r, \theta_{j}$, and canonical momenta $p_{r}, p_{\theta_{j}},(j=1, \ldots, N-1)$. The $N$ hyperspherical coordinates are formed by a radial-type one $r=|\mathbf{q}| \in \mathbb{R}^{+}$and $N-1$ angles $\theta_{j}$ such that $\theta_{k} \in[0,2 \pi)$ for $k<N-1$ and $\theta_{N-1} \in[0, \pi)$. These are defined by

$$
q_{j}=r \cos \theta_{j} \prod_{k=1}^{j-1} \sin \theta_{k}, \quad 1 \leq j<N, \quad q_{N}=r \prod_{k=1}^{N-1} \sin \theta_{k},
$$

where hereafter any product $\prod_{l}^{m}$ such that $l>m$ is assumed to be equal to 1 . The metric (2.3) now adopts the form

$$
\mathrm{d} s^{2}=\left(1+\lambda r^{2}\right)\left(\mathrm{d} r^{2}+r^{2} \mathrm{~d} \Omega^{2}\right),
$$

where $\mathrm{d} \Omega^{2}$ is the metric on the unit $(N-1) \mathrm{D}$ sphere $\mathbb{S}^{N-1}$

$$
\mathrm{d} \Omega^{2}=\sum_{j=1}^{N-1} \mathrm{~d} \theta_{j}^{2} \prod_{k=1}^{j-1} \sin ^{2} \theta_{k}
$$

The relations between $\mathbf{p}$ and $p_{r}, p_{\theta_{j}} \operatorname{read}(1 \leq j<N)$ [2]:

$$
\begin{aligned}
& p_{j}=\prod_{k=1}^{j-1} \sin \theta_{k} \cos \theta_{j} p_{r}+\frac{\cos \theta_{j}}{r} \sum_{l=1}^{j-1} \frac{\prod_{k=l+1}^{j-1} \sin \theta_{k}}{\prod_{m=1}^{l-1} \sin \theta_{m}} \cos \theta_{l} p_{\theta_{l}}-\frac{\sin \theta_{j}}{r \prod_{k=1}^{j-1} \sin \theta_{k}} p_{\theta_{j}}, \\
& p_{N}=\prod_{k=1}^{N-1} \sin \theta_{k} p_{r}+\frac{1}{r} \sum_{l=1}^{N-1} \frac{\prod_{k=l+1}^{N-1} \sin \theta_{k}}{\prod_{m=1}^{l-1} \sin \theta_{m}} \cos \theta_{l} p_{\theta_{l}},
\end{aligned}
$$

where from now on any sum $\sum_{l}^{m}$ such that $l>m$ is assumed to be zero. From 2.9 we obtain that

$$
\mathbf{p}^{2}=p_{r}^{2}+r^{-2} \mathbf{L}^{2}
$$

where $\mathbf{L}^{2}$ is the total angular momentum given by

$$
\mathbf{L}^{2}=\sum_{j=1}^{N-1} p_{\theta_{j}}^{2} \prod_{k=1}^{j-1} \frac{1}{\sin ^{2} \theta_{k}}
$$


By introducing (2.7) and (2.9) in the Hamiltonian (2.2) we find

$$
\mathcal{H}\left(r, p_{r}\right)=\frac{p_{r}^{2}+r^{-2} \mathbf{L}^{2}}{2\left(1+\lambda r^{2}\right)}+\frac{\omega^{2} r^{2}}{2\left(1+\lambda r^{2}\right)}=\mathcal{T}\left(r, p_{r}\right)+\mathcal{U}(r)
$$

The integrals of motion $C_{(m)} \sqrt{2.5}$ adopt a compact form (the remaining $C^{(m)}$ and $I_{i j}$ have more cumbersome expressions):

$$
C_{(m)}=\sum_{j=N-m+1}^{N-1} p_{\theta_{j}}^{2} \prod_{k=N-m+1}^{j-1} \frac{1}{\sin ^{2} \theta_{k}}, \quad m=2, \ldots, N
$$

and $C_{(N)}=\mathbf{L}^{2}$, which is just the second-order Casimir of the $\mathfrak{s o}(N)$-symmetry algebra of a central potential.

Furthermore, the complete integrability determined by the set of $N$ functions $\left\{\mathcal{H}, C_{(m)}\right\}$ $(m=2, \ldots, N)$ leads to a separable set of $N$ equations, since each of them depends on a unique pair of canonical variables. These are the $N-1$ angular equations

$$
\begin{aligned}
& C_{(2)}\left(\theta_{N-1}, p_{\theta_{N-1}}\right)=p_{\theta_{N-1}}^{2}, \\
& C_{(k)}\left(\theta_{N-k+1}, p_{\theta_{N-k+1}}\right)=p_{\theta_{N-k+1}}^{2}+\frac{C_{(k-1)}}{\sin ^{2} \theta_{N-k+1}}, \quad k=3, \ldots, N-1, \\
& C_{(N)}\left(\theta_{1}, p_{\theta_{1}}\right)=p_{\theta_{1}}^{2}+\frac{C_{(N-1)}}{\sin ^{2} \theta_{1}} \equiv \mathbf{L}^{2},
\end{aligned}
$$

together with the single radial equation corresponding to the 1D Hamiltonian 2.12).

\section{The Darboux space and the classical effective potential}

The underlying manifold of the classical Hamiltonian $(2.2)$ is the ND Darboux space with metric (2.3), whose kinetic energy corresponds to the geodesic motion on the complete Riemannian manifold $\mathcal{M}^{N}=\left(\mathbb{R}^{N}, g\right)$ with

$$
g_{i j}:=\left(1+\lambda \mathbf{q}^{2}\right) \delta_{i j}
$$

and provided that $\lambda>0$. The scalar curvature $R(r) \equiv R(|\mathbf{q}|)(2.3)$ coming from this metric is always a negative increasing function such that $\lim _{r \rightarrow \infty} R=0$ and it has a minimum at the origin

$$
R(0)=-2 \lambda N(N-1),
$$

which is exactly the scalar curvature of the ND hyperbolic space with negative constant sectional curvature equal to $-2 \lambda$ (see figure 1). Recall that the four Darboux surfaces are the only $2 \mathrm{D}$ spaces of nonconstant curvature whose geodesic motion is (quadratically) MS, therefore they are the "closest" ones to the classical Riemannian spaces of constant curvature [18, 19].

As far as the nonlinear radial oscillator potential $\mathcal{U}(r) \sqrt[2.12]{ }$ is concerned, we find that it is a positive increasing function of $r$, such that

$$
\mathcal{U}(r)=\frac{\omega^{2} r^{2}}{2\left(1+\lambda r^{2}\right)}, \quad \mathcal{U}(0)=0, \quad \lim _{r \rightarrow \infty} \mathcal{U}(r)=\frac{\omega^{2}}{2 \lambda}
$$




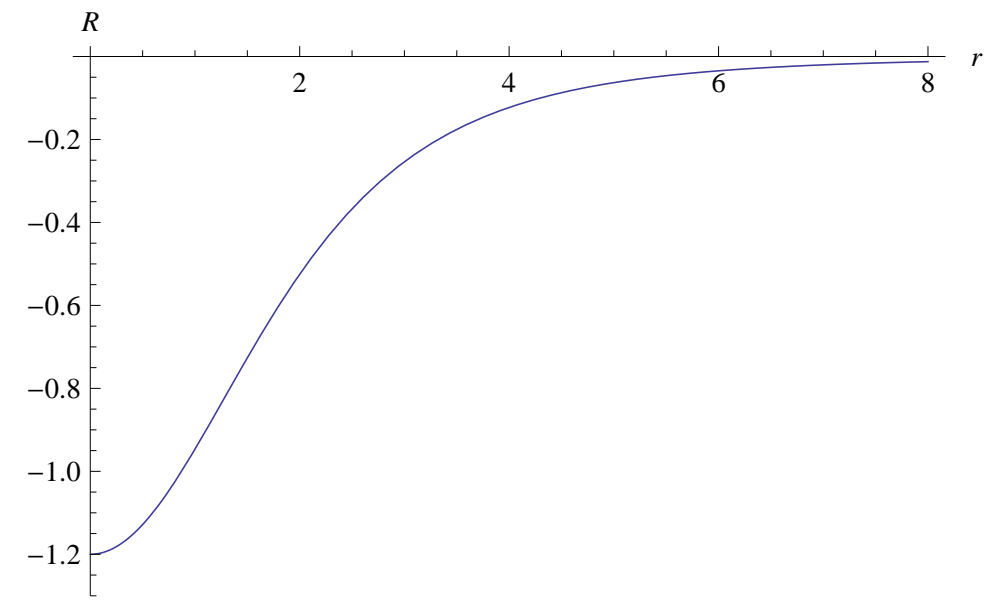

Figure 1: Shape of the scalar curvature (2.3) of the Darboux space where $r=|\mathbf{q}|$ for $N=3$ and $\lambda=0.1$. The minimum is always located at the origin, and its value in this case is $R(0)=-1.2$.

This potential is shown in figure 2 for several values of $\lambda$. Consequently, in contrast with the (Euclidean) isotropic harmonic oscillator, $\mathcal{U}(r)$ yields a nonlinear behavior governed by $\lambda$, which means that the oscillator potential has the asymptotic maximum $\omega^{2} /(2 \lambda)$.

Nevertheless, since the underlying manifold $\mathcal{M}^{N}$ is not flat, the interplay between the oscillator potential $\mathcal{U}(r)$ and the kinetic energy term is rather subtle. For this reason, the complete classical system can be better understood by introducing a classical effective potential. This can be achieved by applying the $1 \mathrm{D}$ canonical transformation defined by

$$
P\left(r, p_{r}\right)=\frac{p_{r}}{\sqrt{1+\lambda r^{2}}}, \quad Q(r)=\frac{1}{2} r \sqrt{1+\lambda r^{2}}+\frac{\operatorname{arcsinh}(\sqrt{\lambda} r)}{2 \sqrt{\lambda}},
$$

(where the new canonical variables fulfill $\{Q, P\}=1$ ), to the radial Hamiltonian (2.12). Notice that $Q(r)$ has a unique (continuously differentiable) inverse $r(Q)$, on the whole positive semiline, that is, both $r, Q \in[0, \infty)$ and $\mathrm{d} Q(r)=\sqrt{1+\lambda r^{2}} \mathrm{~d} r$. In this way, we obtain that

$$
\mathcal{H}(Q, P)=\frac{1}{2} P^{2}+\mathcal{U}_{\mathrm{eff}}(Q), \quad \mathcal{U}_{\mathrm{eff}}(Q(r))=\frac{c_{N}}{2\left(1+\lambda r^{2}\right) r^{2}}+\frac{\omega^{2} r^{2}}{2\left(1+\lambda r^{2}\right)},
$$

where the constant $c_{N} \geq 0$ is the value of the integral of motion corresponding to the square of the total angular momentum $C_{(N)} \equiv \mathbf{L}^{2}(2.13)$. Hence the classical system can be described as a particle on a $1 \mathrm{D}$ flat space under the effective potential $\mathcal{U}_{\text {eff }}(Q(r))$, which is represented in figure 3 .

The analysis of $\mathcal{U}_{\text {eff }}$ shows that this is always positive and it has a minimum located at $r_{\text {min }}$ such that

$$
r_{\min }^{2}=\frac{\lambda c_{N}+\sqrt{\lambda^{2} c_{N}^{2}+\omega^{2} c_{N}}}{\omega^{2}}, \quad \mathcal{U}_{\mathrm{eff}}\left(Q\left(r_{\min }\right)\right)=-\lambda c_{N}+\sqrt{\lambda^{2} c_{N}^{2}+\omega^{2} c_{N}}
$$




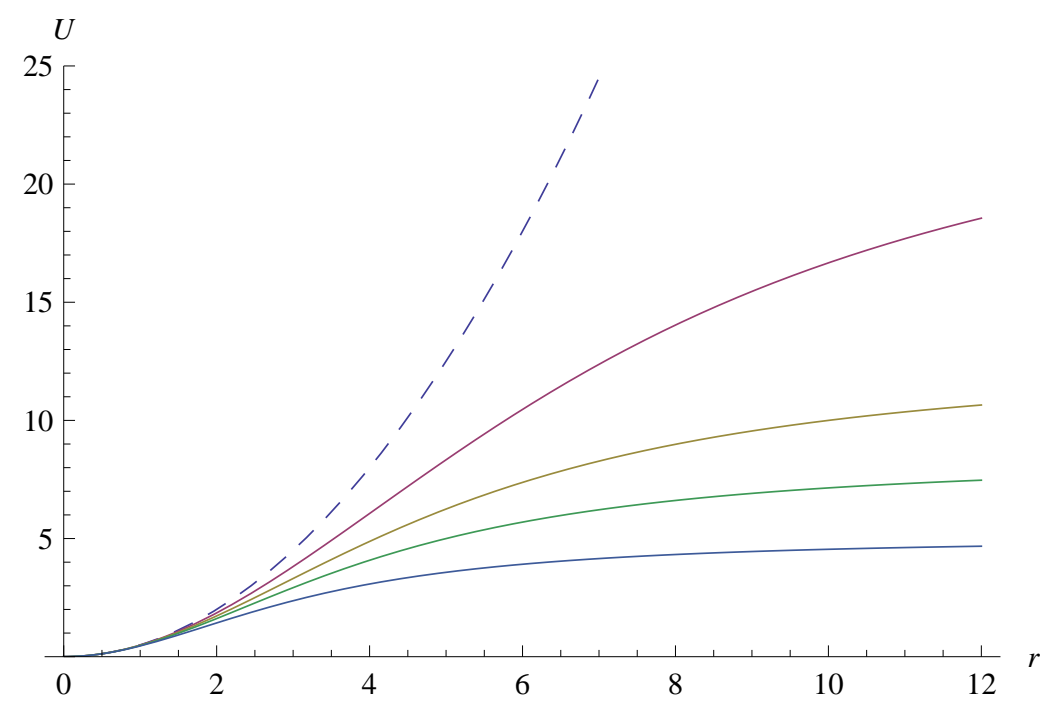

Figure 2: The nonlinear oscillator potential 3.2 with $\omega=1$ for $\lambda=\{0,0.02,0.04,0.06,0.1\}$ starting from the upper dashed line corresponding to the isotropic harmonic oscillator with $\lambda=0$. The limit $r \rightarrow \infty$ gives $\{+\infty, 25,12.5,8.33,5\}$, respectively.

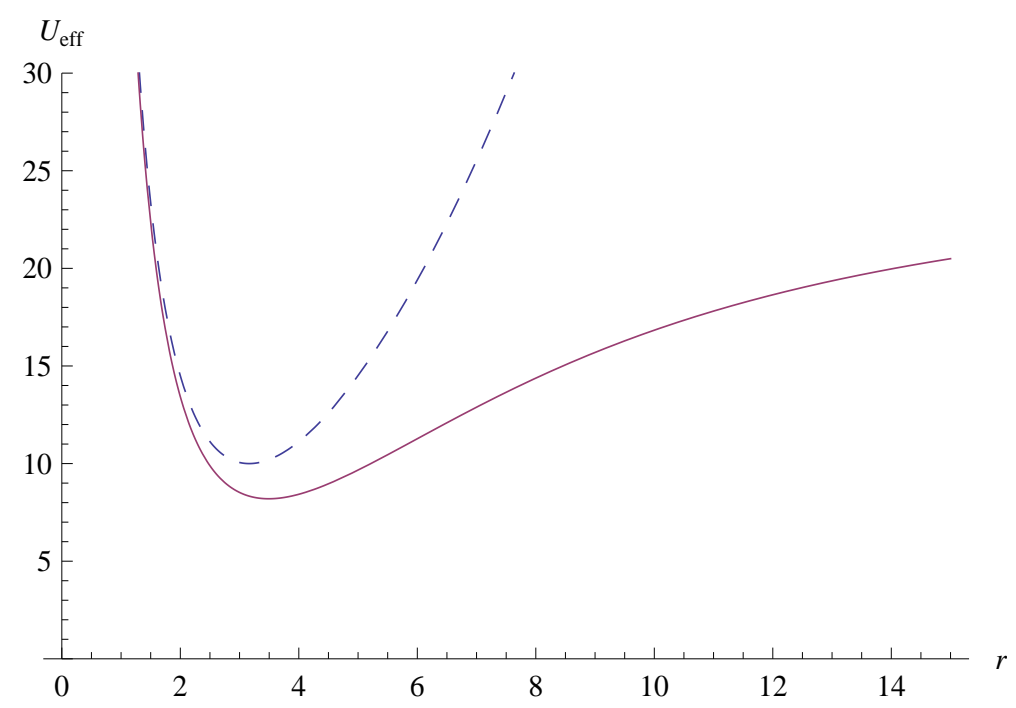

Figure 3: The classical effective nonlinear oscillator potential (3.4) for $\lambda=0.02, c_{N}=100$ and $\omega=1$. The minimum of the potential is located at $r_{\min }=3.49$ with $\mathcal{U}_{\text {eff }}\left(r_{\min }\right)=8.2$ and $\mathcal{U}_{\text {eff }}(\infty)=25$. The dashed line corresponds to the effective potential of the harmonic oscillator with $\lambda=0$ with minimum $\mathcal{U}_{\text {eff }}\left(r_{\min }\right)=10$ at $r_{\min }=3.16$. 
Therefore, $r_{\min }$ and $\mathcal{U}_{\text {eff }}\left(Q\left(r_{\min }\right)\right)$ are, in this order, greater and smaller than those corresponding to the isotropic harmonic oscillator:

$$
\lambda=0: \quad r_{\min }^{2}=\frac{\sqrt{c_{N}}}{\omega}, \quad \mathcal{U}_{\mathrm{eff}}\left(Q\left(r_{\min }\right)\right)=\omega \sqrt{c_{N}}
$$

Moreover $\mathcal{U}_{\text {eff }}$ has two representative limits:

$$
\lim _{r \rightarrow 0} \mathcal{U}_{\mathrm{eff}}(Q(r))=+\infty, \quad \lim _{r \rightarrow \infty} \mathcal{U}_{\mathrm{eff}}(Q(r))=\frac{\omega^{2}}{2 \lambda},
$$

the latter being the same of (3.2). Thus, this effective potential is hydrogen-like and one should expect that its quantum counterpart should have both bounded and unbounded states. The rest of the paper is devoted to solve such a quantum problem in full detail.

\section{Superintegrable quantizations of the Darboux III oscillator}

Let us consider the quantum position and momenta operators, $\hat{\mathbf{q}}, \hat{\mathbf{p}}$, with Lie brackets and differential representation given by

$$
\left[\hat{q}_{i}, \hat{p}_{j}\right]=\mathrm{i} \hbar \delta_{i j}, \quad \hat{q}_{i}=q_{i}, \quad \hat{p}_{i}=-\mathrm{i} \hbar \frac{\partial}{\partial q_{i}} .
$$

Hereafter we will use the standard notation

$$
\nabla=\left(\frac{\partial}{\partial q_{1}}, \ldots, \frac{\partial}{\partial q_{N}}\right), \quad \Delta=\nabla^{2}=\frac{\partial^{2}}{\partial^{2} q_{1}}+\cdots+\frac{\partial^{2}}{\partial^{2} q_{N}} .
$$

\subsection{The Schrödinger quantization}

The so-called "direct" quantization approach can be summarized in the following way [6] (this result is worth to be compared with Theorem 1, but taking into account that hereafter the order of the terms becomes crucial).

Theorem 2. Let $\hat{\mathcal{H}}$ be the quantum Hamiltonian given by

$$
\hat{\mathcal{H}}=\frac{1}{2\left(1+\lambda \hat{\mathbf{q}}^{2}\right)} \hat{\mathbf{p}}^{2}+\frac{\omega^{2} \hat{\mathbf{q}}^{2}}{2\left(1+\lambda \hat{\mathbf{q}}^{2}\right)}=\frac{1}{2\left(1+\lambda \mathbf{q}^{2}\right)}\left(-\hbar^{2} \Delta+\omega^{2} \mathbf{q}^{2}\right) .
$$

Then:

(i) $\hat{\mathcal{H}}$ commutes with the following observables:

- The $(2 N-3)$ quantum angular momentum operators,

$$
\hat{C}^{(m)}=\sum_{1 \leq i<j \leq m}\left(\hat{q}_{i} \hat{p}_{j}-\hat{q}_{j} \hat{p}_{i}\right)^{2}, \quad \hat{C}_{(m)}=\sum_{N-m<i<j \leq N}\left(\hat{q}_{i} \hat{p}_{j}-\hat{q}_{j} \hat{p}_{i}\right)^{2},
$$

where $m=2, \ldots, N$ and $\hat{C}^{(N)}=\hat{C}_{(N)}$.

- The $N^{2}$ operators defining the ND quantum Fradkin tensor, given by

$$
\hat{I}_{i j}=\hat{p}_{i} \hat{p}_{j}-2 \lambda \hat{q}_{i} \hat{q}_{j} \hat{\mathcal{H}}(\hat{\mathbf{q}}, \hat{\mathbf{p}})+\omega^{2} \hat{q}_{i} \hat{q}_{j},
$$


where $i, j=1, \ldots, N$ and such that $\hat{\mathcal{H}}=\frac{1}{2} \sum_{i=1}^{N} \hat{I}_{i i}$.

(ii) Each of the three sets $\left\{\hat{\mathcal{H}}, \hat{C}^{(m)}\right\},\left\{\hat{\mathcal{H}}, \hat{C}_{(m)}\right\}(m=2, \ldots, N)$ and $\left\{\hat{I}_{i i}\right\}(i=1, \ldots, N)$ is formed by $N$ algebraically independent commuting observables.

(iii) The set $\left\{\hat{\mathcal{H}}, \hat{C}^{(m)}, \hat{C}_{(m)}, \hat{I}_{i i}\right\}$ for $m=2, \ldots, N$ with a fixed index $i$ is formed by $2 N-1$ algebraically independent observables.

(iv) $\hat{\mathcal{H}}$ is formally self-adjoint on the $L^{2}$ Hilbert space defined by the scalar product

$$
\langle\Psi \mid \Phi\rangle=\int_{\mathcal{M}^{N}} \overline{\Psi(\mathbf{q})} \Phi(\mathbf{q})\left(1+\lambda \mathbf{q}^{2}\right) \mathrm{d} \mathbf{q}
$$

Proof. Some points of this statement can be straightforwardly proven through the coalgebra symmetry [40, 41, 42] of the quantum Hamiltonian 44.2). Let us consider the $\mathfrak{s l}(2, \mathbb{R})$ Lie coalgebra in the basis $\left\{J_{ \pm}, J_{3}\right\}$ with commutation rules, Casimir invariant and (nondeformed) coproduct given by

$$
\begin{gathered}
{\left[J_{3}, J_{+}\right]=2 \mathrm{i} \hbar J_{+}, \quad\left[J_{3}, J_{-}\right]=-2 \mathrm{i} \hbar J_{-}, \quad\left[J_{-}, J_{+}\right]=4 \mathrm{i} \hbar J_{3},} \\
\mathcal{C}=\frac{1}{2}\left(J_{+} J_{-}+J_{-} J_{+}\right)-J_{3}^{2}, \\
\Delta\left(J_{l}\right)=J_{l} \otimes 1+1 \otimes J_{l}, \quad l=+,-, 3 .
\end{gathered}
$$

An $N$-particle realization of $\mathfrak{s l}(2, \mathbb{R})$ reads

$$
J_{+}=\hat{\mathbf{p}}^{2}, \quad J_{-}=\hat{\mathbf{q}}^{2}, \quad J_{3}=\frac{1}{2}(\hat{\mathbf{q}} \cdot \hat{\mathbf{p}}+\hat{\mathbf{p}} \cdot \hat{\mathbf{q}})=\hat{\mathbf{q}} \cdot \hat{\mathbf{p}}-\frac{1}{2} \mathrm{i} \hbar N .
$$

Therefore, $\hat{\mathcal{H}} 4.2$ has an $\mathfrak{s l}(2, \mathbb{R})$ coalgebra symmetry since it can be written as

$$
\hat{\mathcal{H}}=\frac{1}{2\left(1+\lambda J_{-}\right)} J_{+}+\frac{\omega^{2} J_{-}}{2\left(1+\lambda J_{-}\right)} .
$$

Hence, by construction, $\hat{\mathcal{H}}$ commutes with the $(2 N-3)$ observables $\hat{C}^{(m)}$ and $\hat{C}_{(m)}(m=$ $2, \ldots, N)$ (4.3) which come from the "left" and "right" $m$-th coproducts [41, 42] of the invariant 4.7), respectively, up to an additive constant $\hbar^{2} m(m-4) / 4$. Furthermore, the coalgebra approach also ensures that these are algebraically independent and that each set $\left\{\hat{\mathcal{H}}, \hat{C}^{(m)}\right\}$ and $\left\{\hat{\mathcal{H}}, \hat{C}_{(m)}\right\}$ is formed by $N$ commuting observables (to be more precise, they are polynomially independent as operators in a Jordan algebra).

Next, by direct computations it can be proven that the $N^{2}$ observables $\hat{I}_{i j}$ 4.4 commute with $\hat{\mathcal{H}}$, and that the $N$ (diagonal) observables $\hat{I}_{i i}(i=1, \ldots, N)$ commute amongst themselves as well; it is obvious that the latter $\hat{I}_{i i}$ are algebraically independent. Finally, it is also clear that any single $\hat{I}_{i i}$ is algebraically independent with respect to the set of $2 N-2$ observables $\left\{\hat{\mathcal{H}}, \hat{C}^{(m)}, \hat{C}_{(m)}\right\}$ (as it is when $\left.\lambda=0\right) \square$.

We stress that, as a byproduct of the above proof, any quantum Hamiltonian defined as a function of 4.9 ,

$$
\hat{\mathcal{H}}=\hat{\mathcal{H}}\left(J_{+}, J_{-}, J_{3}\right)=\hat{\mathcal{H}}\left(\hat{\mathbf{p}}^{2}, \hat{\mathbf{q}}^{2}, \hat{\mathbf{q}} \cdot \hat{\mathbf{p}}-\mathrm{i} \hbar N / 2\right),
$$

is endowed with the same $\mathfrak{s l}(2, \mathbb{R})$ coalgebra symmetry. This shows that this is quasi-MS [2, 41, 42, that is, it commutes, at least, with the $(2 N-3)$ observables $\hat{C}^{(m)}$ and $\hat{C}_{(m)}$. In this 
respect, we remark that what makes the quantum Darboux III oscillator 4.10 very special, is the existence of a quantum Fradkin tensor formed by the "additional" symmetries $\hat{I}_{i j}$. This algebraic property implies that the system is MS and, as we shall see, that its discrete energy spectrum is maximally degenerate.

\subsection{The Laplace-Beltrami quantization}

When dealing with curved spaces with metric and classical kinetic term given by

$$
\mathrm{d} s^{2}=\sum_{i, j=1}^{N} g_{i j}(\mathbf{q}) \mathrm{d} q_{i} \mathrm{~d} q_{j}, \quad \mathcal{T}(\mathbf{q}, \mathbf{p})=\frac{1}{2} \sum_{i, j=1}^{N} g^{i j}(\mathbf{q}) p_{i} p_{j}
$$

the LB operator

$$
\Delta_{\mathrm{LB}}=\sum_{i, j=1}^{N} \frac{1}{\sqrt{g}} \partial_{i} \sqrt{g} g^{i j} \partial_{j},
$$

can be used in order to define the quantum kinetic energy as

$$
\hat{\mathcal{T}}_{\mathrm{LB}}(\hat{\mathbf{q}}, \hat{\mathbf{p}})=-\frac{\hbar^{2}}{2} \Delta_{\mathrm{LB}}
$$

where $g^{i j}$ is the inverse of the metric tensor $g_{i j}$ and $g$ is its determinant (see, for instance, [19, 43 ). If we apply such LB quantization to the Hamiltonian (2.2) with metric tensor (3.1) we get

$$
\begin{aligned}
\hat{\mathcal{H}}_{\mathrm{LB}} & =-\frac{\hbar^{2}}{2} \Delta_{\mathrm{LB}}+\frac{\omega^{2} \mathbf{q}^{2}}{2\left(1+\lambda \mathbf{q}^{2}\right)} \\
& =-\frac{\hbar^{2}}{2\left(1+\lambda \mathbf{q}^{2}\right)} \Delta-\frac{\hbar^{2} \lambda(N-2)}{2\left(1+\lambda \mathbf{q}^{2}\right)^{2}}(\mathbf{q} \cdot \nabla)+\frac{\omega^{2} \mathbf{q}^{2}}{2\left(1+\lambda \mathbf{q}^{2}\right)} .
\end{aligned}
$$

Then, $\hat{\mathcal{H}} 4.2$ and $\hat{\mathcal{H}}_{\mathrm{LB}}$ only coincide in the case $N=2$ (as it should be for any sperically symmetric space [39]) and for $N>2$ they differ by a momentum-dependent potential, namely:

$$
\hat{\mathcal{H}}_{\mathrm{LB}}=\hat{\mathcal{H}}+\mathcal{U}_{1}, \quad \mathcal{U}_{1}(\hat{\mathbf{q}}, \hat{\mathbf{p}})=-\mathrm{i} \frac{\hbar \lambda(N-2)}{2\left(1+\lambda \hat{\mathbf{q}}^{2}\right)^{2}}(\hat{\mathbf{q}} \cdot \hat{\mathbf{p}})
$$

where we have introduced the quantum variables (4.1). Notice that $\mathcal{U}_{1}$ is linear in $\hbar$, so this term does not have any classical analog. This situation reminds what happens in the context of the so-called quasi-exactly solvable quantum models [44. On the other hand, although the Hamiltonian $\hat{\mathcal{H}}_{\mathrm{LB}}$ commutes with the operators 4.3 (the quantum correction $\mathcal{U}_{1}$ preserves the $\mathfrak{s l}(2, \mathbb{R})$ coalgebra symmetry $(4.6)-(4.9)$ ), in this case there is no hint about the existence of an additional symmetry of the type (4.4).

Nevertheless, it is possible to find a "superintegrable" LB quantization (in the sense that it does preserve the MS property) by adding a second potential term to $\hat{\mathcal{H}}$ (besides $\mathcal{U}_{1}$ ) thus conveying $N^{2}$ additional integrals of the type (4.4) together with the separability property 
in terms of the $N$ "diagonal" ones. In order to achieve this result we will relate $\hat{\mathcal{H}}$ and $\hat{\mathcal{H}}_{\mathrm{LB}}$ through a similarity transformation. If we apply $\hat{\mathcal{H}}_{\mathrm{LB}}$ to the product $\exp (f(\mathbf{q})) \Psi(\mathbf{q})$ we get:

$$
\begin{aligned}
\hat{\mathcal{H}}_{\mathrm{LB}} \mathrm{e}^{f} \Psi= & -\frac{\hbar^{2} \mathrm{e}^{f}}{2\left(1+\lambda \mathbf{q}^{2}\right)} \Delta \Psi-\frac{\hbar^{2} \mathrm{e}^{f}}{1+\lambda \mathbf{q}^{2}}(\nabla f \cdot \nabla \Psi)-\frac{\hbar^{2} \mathrm{e}^{f}}{2\left(1+\lambda \mathbf{q}^{2}\right)}\left(\Delta f+(\nabla f)^{2}\right) \Psi \\
& -\frac{\hbar^{2} \lambda(N-2) \mathrm{e}^{f}}{2\left(1+\lambda \mathbf{q}^{2}\right)^{2}}(\mathbf{q} \cdot \nabla \Psi)-\frac{\hbar^{2} \lambda(N-2) \mathrm{e}^{f}}{2\left(1+\lambda \mathbf{q}^{2}\right)^{2}}(\mathbf{q} \cdot \nabla f) \Psi+\frac{\omega \mathbf{q}^{2}}{2\left(1+\lambda \mathbf{q}^{2}\right)} \mathrm{e}^{f} \Psi .
\end{aligned}
$$

The two terms depending on $(\nabla f \cdot \nabla \Psi)$ and $(\mathbf{q} \cdot \nabla \Psi)$ can be removed by setting

$$
f(\mathbf{q})=\frac{2-N}{4} \ln \left(1+\lambda \mathbf{q}^{2}\right),
$$

which, in turn, means that there is a similarity transformation connecting $\hat{\mathcal{H}}$ and $\hat{\mathcal{H}}_{\mathrm{LB}}$ :

$$
\begin{aligned}
& \hat{\mathcal{H}}_{\mathrm{LB}} \mathrm{e}^{f} \Psi=\mathrm{e}^{f}\left(\hat{\mathcal{H}}-\mathcal{U}_{2}(\mathbf{q})\right) \Psi, \quad \hat{\mathcal{H}}_{\mathrm{LB}}=\mathrm{e}^{f} \hat{\mathcal{H}} \mathrm{e}^{-f}-\mathcal{U}_{2}, \\
& \mathcal{U}_{2}(\mathbf{q})=-\frac{\hbar^{2} \lambda(N-2)}{8\left(1+\lambda \mathbf{q}^{2}\right)^{3}}\left(2 N+3 \lambda \mathbf{q}^{2}(N-2)\right) .
\end{aligned}
$$

Notice that the multiplication operator $\mathrm{e}^{f}$ defines a transformation mapping

$$
L^{2}\left(\mathcal{M}^{N}\right)=\left(\mathbb{R}^{N},\left(1+\lambda \mathbf{q}^{2}\right) \mathrm{d} \mathbf{q}\right) \quad \text { into } \quad L^{2}\left(\mathcal{M}^{N}\right)=L^{2}\left(\mathbb{R}^{N},\left(1+\lambda \mathbf{q}^{2}\right)^{N / 2} \mathrm{~d} \mathbf{q}\right),
$$

which is the natural $L^{2}$ space defined by the Riemannian metric. We remark that either for $N=2$ or when $\lambda=0, f(\mathbf{q})=\mathcal{U}_{2}(\mathbf{q})=0$, and notice also that the central potential $\mathcal{U}_{2}$ is a pure quantum term as it depends on $\hbar^{2}$. The latter result suggests to consider a "transformed-LB" Hamiltonian defined by

$$
\hat{\mathcal{H}}_{\mathrm{TLB}}=\hat{\mathcal{H}}_{\mathrm{LB}}+\mathcal{U}_{2}=\hat{\mathcal{H}}+\mathcal{U}_{1}+\mathcal{U}_{2}
$$

which satisfies

$$
\hat{\mathcal{H}}_{\mathrm{TLB}}=\mathrm{e}^{f} \hat{\mathcal{H}} \mathrm{e}^{-f} .
$$

Hence, as a direct consequence, all the symmetries of $\hat{\mathcal{H}}$ give rise to those corresponding to $\hat{\mathcal{H}}_{\text {TLB }}$ :

$$
\hat{X}_{\mathrm{TLB}}=\mathrm{e}^{f} \hat{X} \mathrm{e}^{-f}, \quad \hat{X}=\left\{\hat{C}^{(m)}, \hat{C}_{(m)}, \hat{I}_{i j}\right\}, \quad\left[\hat{\mathcal{H}}_{\mathrm{TLB}}, \hat{X}_{\mathrm{TLB}}\right]=0 .
$$

Therefore, by taking into account Theorem 2 and the equations 4.15 and 4.16 ) we find that $\hat{\mathcal{H}}_{\mathrm{TLB}}$ is, in fact, a quantum MS Hamiltonian.

Theorem 3. Let $\hat{\mathcal{H}}_{\mathrm{TLB}}$ be the quantum Hamiltonian given by

$$
\begin{aligned}
\hat{\mathcal{H}}_{\mathrm{TLB}}= & \frac{1}{2\left(1+\lambda \hat{\mathbf{q}}^{2}\right)} \hat{\mathbf{p}}^{2}+\frac{\omega^{2} \hat{\mathbf{q}}^{2}}{2\left(1+\lambda \hat{\mathbf{q}}^{2}\right)}-\mathrm{i} \frac{\hbar \lambda(N-2)}{2\left(1+\lambda \hat{\mathbf{q}}^{2}\right)^{2}}(\hat{\mathbf{q}} \cdot \hat{\mathbf{p}}) \\
& -\frac{\hbar^{2} \lambda(N-2)}{8\left(1+\lambda \hat{\mathbf{q}}^{2}\right)^{3}}\left(2 N+3 \lambda \hat{\mathbf{q}}^{2}(N-2)\right) \\
= & -\frac{\hbar^{2}}{2} \Delta_{\mathrm{LB}}+\frac{\omega^{2} \mathbf{q}^{2}}{2\left(1+\lambda \mathbf{q}^{2}\right)}-\frac{\hbar^{2} \lambda(N-2)}{8\left(1+\lambda \mathbf{q}^{2}\right)^{3}}\left(2 N+3 \lambda \mathbf{q}^{2}(N-2)\right) .
\end{aligned}
$$


Then:

(i) $\hat{\mathcal{H}}_{\mathrm{TLB}}$ commutes with the same observables 4.3$)$, that is, $\hat{C}_{\mathrm{TLB}}^{(m)}=\hat{C}^{(m)}$ and $\hat{C}_{\mathrm{TLB},(m)}=$ $\hat{C}_{(m)}$, as well as with the $N^{2}$ Fradkin operators given by

$$
\begin{gathered}
\hat{I}_{\mathrm{TLB}, i j}=\hat{p}_{i} \hat{p}_{j}-(N-2) \frac{\mathrm{i} \hbar \lambda}{2\left(1+\lambda \hat{\mathbf{q}}^{2}\right)}\left(\hat{q}_{i} \hat{p}_{j}+\hat{q}_{j} \hat{p}_{i}\right)+\frac{(N-2) \hbar^{2} \lambda^{2} \hat{q}_{i} \hat{q}_{j}}{\left(1+\lambda \hat{\mathbf{q}}^{2}\right)^{2}}\left(1-\frac{N-2}{4}\right) \\
-\frac{(N-2) \hbar^{2} \lambda}{2\left(1+\lambda \hat{\mathbf{q}}^{2}\right)} \delta_{i j}-2 \lambda \hat{q}_{i} \hat{q}_{j} \hat{\mathcal{H}}_{\mathrm{TLB}}(\hat{\mathbf{q}}, \hat{\mathbf{p}})+\omega^{2} \hat{q}_{i} \hat{q}_{j},
\end{gathered}
$$

with $i, j=1, \ldots, N$ and such that $\hat{\mathcal{H}}_{\mathrm{TLB}}=\frac{1}{2} \sum_{i=1}^{N} \hat{I}_{\mathrm{TLB}, i i}$.

(ii) Each of the three sets $\left\{\hat{\mathcal{H}}_{\mathrm{TLB}}, \hat{C}^{(m)}\right\},\left\{\hat{\mathcal{H}}_{\mathrm{TLB}}, \hat{C}_{(m)}\right\}(m=2, \ldots, N)$ and $\left\{\hat{I}_{\mathrm{TLB}, i i}\right\} \quad(i=$ $1, \ldots, N)$ is formed by $N$ algebraically independent commuting observables.

(iii) The set $\left\{\hat{\mathcal{H}}_{\mathrm{TLB}}, \hat{C}^{(m)}, \hat{C}_{(m)}, \hat{I}_{\mathrm{TLB}, i i}\right\}$ for $m=2, \ldots, N$ with a fixed index $i$ is formed by $2 N-1$ algebraically independent observables.

(iv) $\hat{\mathcal{H}}_{\mathrm{TLB}}$ is formally self-adjoint on the space $L^{2}\left(\mathcal{M}^{N}\right)$ associated with the underlying Darboux space, defined by

$$
\langle\Psi \mid \Phi\rangle_{\mathrm{TLB}}=\int_{\mathcal{M}^{N}} \overline{\Psi(\mathbf{q})} \Phi(\mathbf{q})\left(1+\lambda \mathbf{q}^{2}\right)^{N / 2} \mathrm{~d} \mathbf{q} .
$$

Therefore, according to the above statement, $\hat{\mathcal{H}}_{\mathrm{TLB}}$ can be seen as the appropriate LBquantization of the classical Hamiltonian $(2.2)$ as it manifestly preserves the MS property. Such a quantization requires to add a linear momentum-dependent potential $\mathcal{U}_{1}$ (coming from the quantum kinetic energy) plus an "additional" central potential $\mathcal{U}_{2}$ (coming from the MS property) to the Hamiltonian $\hat{\mathcal{H}}$. Clearly, the eigenfunctions of $\hat{\mathcal{H}}_{\mathrm{TLB}}$ can be read off from those of $\hat{\mathcal{H}}$ by means of 4.15 .

Remark. Interestingly, the quantum correction $\mathcal{U}_{2}(4.13)$ to the oscillator potential arising from the similarity transformation 4.12 is proportional to the scalar curvature of the underlying metric $(2.3)$ :

$$
\mathcal{U}_{2}=\frac{\hbar^{2}(N-2)}{8(N-1)} R
$$

which vanishes for any 2D space and in the (most frequently studied) case of spaces of constant curvature gives simply an additional constant. Therefore, the transformed-LB quantization prescription is equivalent to imposing that

$$
\hat{\mathcal{H}}_{\mathrm{TLB}}=-\frac{\hbar^{2}}{2} \Delta_{\mathrm{c}}+\mathcal{U}(\mathbf{q}),
$$

that is, to asserting that the appropriate quantum kinetic energy operator is essentially the conformal Laplacian (see, for instance, [10])

$$
\Delta_{\mathrm{c}}=\Delta_{\mathrm{LB}}-\frac{(N-2)}{4(N-1)} R,
$$

rather than the ordinary Laplacian (or LB operator). This is in full agreement with many prescriptions used in the analysis of scalar field theories in General Relativity or when dealing with quantization on arbitrary Riemannian manifolds [7, 8, 9]. However, we are not aware of any other instances where the convenience of this prescription has been motivated by superintegrability arguments. 


\subsection{A position-dependent mass quantization}

In the framework of PDM Hamiltonian systems there are also several ways to define the quantum kinetic energy term. A general approach depending on three parameters subjected to a constraint can be found in [14] (see also [16]). We shall consider here the proposal given in [15] and based on Galilean invariance arguments, which is the one extensively used in the condensed matter literature [12, 13]. Such a PDM quantization, $\mathcal{T}(\mathbf{q}, \mathbf{p}) \rightarrow \hat{\mathcal{T}}_{\mathrm{PDM}}(\hat{\mathbf{q}}, \hat{\mathbf{p}})$, is defined as

$$
\hat{\mathcal{T}}_{\mathrm{PDM}}(\hat{\mathbf{q}}, \hat{\mathbf{p}})=\frac{1}{2} \hat{\mathbf{p}} \cdot \frac{1}{\left(1+\lambda \hat{\mathbf{q}}^{2}\right)} \hat{\mathbf{p}}=-\frac{\hbar^{2}}{2} \nabla \cdot \frac{1}{\left(1+\lambda \mathbf{q}^{2}\right)} \nabla .
$$

Then, by adding the oscillator potential and ordering terms in the kinetic term, we obtain the following PDM quantization of the Hamiltonian (2.2):

$$
\begin{aligned}
\hat{\mathcal{H}}_{\mathrm{PDM}} & =\hat{\mathcal{T}}_{\mathrm{PDM}}(\hat{\mathbf{q}}, \hat{\mathbf{p}})+\mathcal{U}(\hat{\mathbf{q}}) \\
& =-\frac{\hbar^{2}}{2\left(1+\lambda \mathbf{q}^{2}\right)} \Delta+\frac{\hbar^{2} \lambda}{\left(1+\lambda \mathbf{q}^{2}\right)^{2}}(\mathbf{q} \cdot \nabla)+\frac{\omega^{2} \mathbf{q}^{2}}{2\left(1+\lambda \mathbf{q}^{2}\right)} .
\end{aligned}
$$

Hence the difference between $\hat{\mathcal{H}} \sqrt{4.2}$ and $\hat{\mathcal{H}}_{\text {PDM }}$ relies again in a momentum-dependent potential,

$$
\hat{\mathcal{H}}_{\mathrm{PDM}}=\hat{\mathcal{H}}+\mathcal{V}_{1}, \quad \mathcal{V}_{1}(\hat{\mathbf{q}}, \hat{\mathbf{p}})=\mathrm{i} \frac{\hbar \lambda}{\left(1+\lambda \hat{\mathbf{q}}^{2}\right)^{2}}(\hat{\mathbf{q}} \cdot \hat{\mathbf{p}}) .
$$

Similarly to the LB quantization, the MS property can be explicitly restored through a similarity transformation and this process will require to add another central potential to the initial $\hat{\mathcal{H}}_{\mathrm{PDM}}$.

Explicitly, if we apply $\hat{\mathcal{H}}_{\mathrm{PDM}}$ to the product $\exp (v(\mathbf{q})) \Psi(\mathbf{q})$ and define

$$
v(\mathbf{q})=\frac{1}{2} \ln \left(1+\lambda \mathbf{q}^{2}\right)
$$

then we get the following similarity transformation between $\hat{\mathcal{H}}$ and $\hat{\mathcal{H}}_{\mathrm{PDM}}$ :

$$
\begin{aligned}
& \hat{\mathcal{H}}_{\mathrm{PDM}^{v}} \Psi=\mathrm{e}^{v}\left(\hat{\mathcal{H}}-\mathcal{V}_{2}(\mathbf{q})\right) \Psi, \quad \hat{\mathcal{H}}_{\mathrm{PDM}}=\mathrm{e}^{v} \hat{\mathcal{H}} \mathrm{e}^{-v}-\mathcal{V}_{2}, \\
& \mathcal{V}_{2}(\mathbf{q})=\frac{\hbar^{2} \lambda}{2\left(1+\lambda \mathbf{q}^{2}\right)^{3}}\left(N+\lambda \mathbf{q}^{2}(N-3)\right)
\end{aligned}
$$

Hence, in contrast with the LB quantization, now both $v(\mathbf{q})$ and $\mathcal{V}_{2}(\mathbf{q})$ are nontrivial for any dimension $N$ (including $N=2$ ). In this way, we define the following "transformed-PDM" Hamiltonian,

$$
\hat{\mathcal{H}}_{\mathrm{TPDM}}=\hat{\mathcal{H}}_{\mathrm{PDM}}+\mathcal{V}_{2}=\hat{\mathcal{H}}+\mathcal{V}_{1}+\mathcal{V}_{2}, \quad \hat{\mathcal{H}}_{\mathrm{TPDM}}=\mathrm{e}^{v} \hat{\mathcal{H}} \mathrm{e}^{-v}
$$

whose symmetries are thus obtained from those of $\hat{\mathcal{H}}$ as

$$
\hat{X}_{\mathrm{TPDM}}=\mathrm{e}^{v} \hat{X} \mathrm{e}^{-v}, \quad \hat{X}=\left\{\hat{C}^{(m)}, \hat{C}_{(m)}, \hat{I}_{i j}\right\}, \quad\left[\hat{\mathcal{H}}_{\mathrm{TPDM}}, \hat{X}_{\mathrm{TPDM}}\right]=0 .
$$

The MS property of the Hamiltonian $\hat{H}_{\mathrm{TPDM}}$ is summarized in the following statement. 
Theorem 4. Let $\hat{\mathcal{H}}_{\mathrm{TPDM}}$ be the quantum Hamiltonian defined by

$$
\hat{\mathcal{H}}_{\mathrm{TPDM}}=\frac{1}{2\left(1+\lambda \hat{\mathbf{q}}^{2}\right)} \hat{\mathbf{p}}^{2}+\frac{\omega^{2} \hat{\mathbf{q}}^{2}}{2\left(1+\lambda \hat{\mathbf{q}}^{2}\right)}+\frac{\mathrm{i} \hbar \lambda}{\left(1+\lambda \hat{\mathbf{q}}^{2}\right)^{2}}(\hat{\mathbf{q}} \cdot \hat{\mathbf{p}})+\frac{\hbar^{2} \lambda\left(N+\lambda \mathbf{q}^{2}(N-3)\right)}{2\left(1+\lambda \mathbf{q}^{2}\right)^{3}} .
$$

Then:

(i) $\hat{\mathcal{H}}_{\mathrm{TPDM}}$ commutes with the observables (4.3) as well as with $(i, j=1, \ldots, N)$

$$
\begin{aligned}
\hat{I}_{\mathrm{TPDM}, i j}= & \hat{p}_{i} \hat{p}_{j}+\frac{\mathrm{i} \hbar \lambda}{\left(1+\lambda \hat{\mathbf{q}}^{2}\right)}\left(\hat{q}_{i} \hat{p}_{j}+\hat{q}_{j} \hat{p}_{i}\right)+\frac{\hbar^{2} \lambda}{\left(1+\lambda \hat{\mathbf{q}}^{2}\right)}\left(\delta_{i j}-\frac{3 \lambda \hat{q}_{i} \hat{q}_{j}}{\left(1+\lambda \hat{\mathbf{q}}^{2}\right)}\right) \\
& -2 \lambda \hat{q}_{i} \hat{q}_{j} \hat{\mathcal{H}}_{\mathrm{TPDM}}(\hat{\mathbf{q}}, \hat{\mathbf{p}})+\omega^{2} \hat{q}_{i} \hat{q}_{j},
\end{aligned}
$$

which form a quantum Fradkin tensor and verifiy that $\hat{\mathcal{H}}_{\mathrm{TPDM}}=\frac{1}{2} \sum_{i=1}^{N} \hat{I}_{\mathrm{TPDM}, i i}$.

(ii) Each of the three sets $\left\{\hat{\mathcal{H}}_{\mathrm{TPDM}}, \hat{C}^{(m)}\right\},\left\{\hat{\mathcal{H}}_{\mathrm{TPDM}}, \hat{C}_{(m)}\right\}(m=2, \ldots, N)$ and $\left\{\hat{I}_{\mathrm{TPDM}, i i}\right\}$ $(i=1, \ldots, N)$ is formed by $N$ algebraically independent commuting observables.

(iii) The set $\left\{\hat{\mathcal{H}}_{\mathrm{TPDM}}, \hat{C}^{(m)}, \hat{C}_{(m)}, \hat{I}_{\mathrm{TPDM}, i i}\right\}$ for $m=2, \ldots, N$ with a fixed index $i$ is formed by $2 N-1$ algebraically independent observables.

(iv) $\hat{\mathcal{H}}_{\mathrm{TPDM}}$ is formally self-adjoint on the standard $L^{2}$ space with product

$$
\langle\Psi \mid \Phi\rangle_{\mathrm{TPDM}}=\int_{\mathcal{M}^{N}} \overline{\Psi(\mathbf{q})} \Phi(\mathbf{q}) \mathrm{d} \mathbf{q} .
$$

Finally, we remark that by combining the similarity transformations 4.15 and 4.20 we obtain the relationship between $\hat{\mathcal{H}}_{\mathrm{TLB}}$ and $\hat{\mathcal{H}}_{\mathrm{TPDM}}$ :

$$
\hat{\mathcal{H}}_{\mathrm{TPDM}}=\mathrm{e}^{v-f} \hat{\mathcal{H}}_{\mathrm{TLB}} \mathrm{e}^{-(v-f)}=\left(1+\lambda \mathbf{q}^{2}\right)^{N / 4} \hat{\mathcal{H}}_{\mathrm{TLB}}\left(1+\lambda \mathbf{q}^{2}\right)^{-N / 4} .
$$

\section{$5 \quad$ Radial Schrödinger equations}

In this section we obtain the $1 \mathrm{D}$ radial Schrödinger equation coming from each of the above three ND quantum Hamiltonians by, firstly, introducing hyperspherical coordinates and, secondly, by making use of the observables $\hat{C}_{(m)} 4.3$ that encode the full spherical symmetry of the three systems.

Let us introduce the map from the initial quantum operators 4.1 to the quantum hyperspherical ones $\hat{r}, \hat{\theta}_{j}, \hat{p}_{r}, \hat{p}_{\theta_{j}}(j=1 \ldots, N-1)$ with Lie brackets and differential representation given by

$$
\begin{aligned}
& {\left[\hat{r}, \hat{p}_{r}\right]=\mathrm{i} \hbar, \quad\left[\hat{r}, \hat{p}_{\theta_{j}}\right]=0, \quad\left[\hat{\theta}_{j}, \hat{p}_{r}\right]=0, \quad\left[\hat{\theta}_{j}, \hat{p}_{\theta_{k}}\right]=\mathrm{i} \hbar \delta_{j k},} \\
& \hat{r}=r, \quad \hat{p}_{r}=-\mathrm{i} \hbar \frac{\partial}{\partial r}, \quad \hat{\theta}_{j}=\theta_{j}, \quad \hat{p}_{\theta_{k}}=-\mathrm{i} \hbar \frac{\partial}{\partial \theta_{j}} .
\end{aligned}
$$

Here we point out that the "radial and phase operators" that we have just introduced are nothing but formal multiplicative operators on the angular variables, whose "canonical" trans- 
formation rules with respect to the Cartesian ones are:

$$
\begin{aligned}
& \hat{q}_{j}=\hat{r} \cos \hat{\theta}_{j} \prod_{k=1}^{j-1} \sin \hat{\theta}_{k}, \quad 1 \leq j<N ; \quad \hat{q}_{N}=\hat{r} \prod_{k=1}^{N-1} \sin \hat{\theta}_{k}, \\
& \hat{p}_{j}=\prod_{k=1}^{j-1} \sin \hat{\theta}_{k} \cos \hat{\theta}_{j} \hat{p}_{r}+\frac{\cos \hat{\theta}_{j}}{\hat{r}} \sum_{l=1}^{j-1} \frac{\prod_{k=l+1}^{j-1} \sin \hat{\theta}_{k}}{\prod_{m=1}^{l-1} \sin \hat{\theta}_{m}} \cos \hat{\theta}_{l} \hat{p}_{\theta_{l}}-\frac{\sin \hat{\theta}_{j}}{\hat{r} \prod_{k=1}^{j-1} \sin \hat{\theta}_{k}} \hat{p}_{\theta_{j}}, \\
& \hat{p}_{N}=\prod_{k=1}^{N-1} \sin \hat{\theta}_{k} \hat{p}_{r}+\frac{1}{\hat{r}} \sum_{l=1}^{N-1} \frac{\prod_{k=l+1}^{N-1} \sin \hat{\theta}_{k}}{\prod_{m=1}^{l-1} \sin \hat{\theta}_{m}} \cos \hat{\theta}_{l} \hat{p}_{\theta_{l}} .
\end{aligned}
$$

Hence we obtain that

$$
\hat{\mathbf{q}}^{2}=\hat{r}^{2}, \quad \hat{\mathbf{p}}^{2}=\frac{1}{\hat{r}^{N-1}} \hat{p}_{r} \hat{r}^{N-1} \hat{p}_{r}+\frac{\hat{\mathbf{L}}^{2}}{\hat{r}^{2}}=\hat{p}_{r}^{2}-\mathrm{i} \hbar \frac{(N-1)}{\hat{r}} \hat{p}_{r}+\frac{\hat{\mathbf{L}}^{2}}{\hat{r}^{2}}, \quad \hat{\mathbf{q}} \cdot \hat{\mathbf{p}}=\hat{r} \hat{p}_{r},
$$

where $\hat{\mathbf{L}}^{2}$ is the square of the total quantum angular momentum given by

$$
\hat{\mathbf{L}}^{2}=\sum_{j=1}^{N-1}\left(\prod_{k=1}^{j-1} \frac{1}{\sin ^{2} \hat{\theta}_{k}}\right) \frac{1}{\left(\sin \hat{\theta}_{j}\right)^{N-1-j}} \hat{p}_{\theta_{j}}\left(\sin \hat{\theta}_{j}\right)^{N-1-j} \hat{p}_{\theta_{j}} .
$$

Notice that the expressions $(5.2)$ provide a $1 \mathrm{D}$ (radial) representation of the $\mathfrak{s l}(2, \mathbb{R})$ Lie algebra 4.6 by introducing them in 4.9.

The $N-1$ commuting observables $\hat{C}_{(m)}$ 4.3) turn out to be $(m=2, \ldots, N)$

$$
\hat{C}_{(m)}=\sum_{j=N-m+1}^{N-1}\left(\prod_{k=N-m+1}^{j-1} \frac{1}{\sin ^{2} \hat{\theta}_{k}}\right) \frac{1}{\left(\sin \hat{\theta}_{j}\right)^{N-1-j}} \hat{p}_{\theta_{j}}\left(\sin \hat{\theta}_{j}\right)^{N-1-j} \hat{p}_{\theta_{j}},
$$

with $\hat{C}_{(N)}=\hat{\mathbf{L}}^{2}$. Thus we obtain a set of $N-1$ angular equations $(k=3, \ldots, N-1)$ :

$$
\begin{aligned}
& \hat{C}_{(2)}\left(\hat{\theta}_{N-1}, \hat{p}_{\theta_{N-1}}\right)=\hat{p}_{\theta_{N-1}}^{2}, \\
& \hat{C}_{(k)}\left(\hat{\theta}_{N-k+1}, \hat{p}_{\theta_{N-k+1}}\right)=\frac{1}{\left(\sin \hat{\theta}_{N-k+1}\right)^{k-2}} \hat{p}_{\theta_{N-k+1}}\left(\sin \hat{\theta}_{N-k+1}\right)^{k-2} \hat{p}_{\theta_{N-k+1}}+\frac{\hat{C}_{(k-1)}}{\sin ^{2} \hat{\theta}_{N-k+1}}, \\
& \hat{C}_{(N)}\left(\hat{\theta}_{1}, \hat{p}_{\theta_{1}}\right)=\frac{1}{\left(\sin \hat{\theta}_{1}\right)^{N-2}} \hat{p}_{\theta_{1}}\left(\sin \hat{\theta}_{1}\right)^{N-2} \hat{p}_{\theta_{1}}+\frac{\hat{C}_{(N-1)}}{\sin ^{2} \hat{\theta}_{1}} \equiv \hat{\mathbf{L}}^{2},
\end{aligned}
$$

which are worth to be compared with (2.13). Therefore the quantum radial Hamiltonian corresponding to 4.2 is obtained in the form

$$
\hat{\mathcal{H}}\left(\hat{r}, \hat{p}_{r}\right)=\frac{1}{2\left(1+\lambda \hat{r}^{2}\right)}\left(\frac{1}{\hat{r}^{N-1}} \hat{p}_{r} \hat{r}^{N-1} \hat{p}_{r}+\frac{\hat{\mathbf{L}}^{2}}{\hat{r}^{2}}+\omega^{2} \hat{r}^{2}\right) .
$$

After reordering terms and introducing the differential operators (5.1) in the Hamiltonian 5.4 we arrive at the following Schrödinger equation, $\hat{\mathcal{H}} \Psi=E \Psi$,

$$
\frac{1}{2\left(1+\lambda r^{2}\right)}\left(-\hbar^{2} \partial_{r}^{2}-\frac{\hbar^{2}(N-1)}{r} \partial_{r}+\frac{\hat{\mathbf{L}}^{2}}{r^{2}}+\omega^{2} r^{2}\right) \Psi(r, \boldsymbol{\theta})=E \Psi(r, \boldsymbol{\theta}),
$$


where $\boldsymbol{\theta}=\left(\theta_{1}, \ldots, \theta_{N-1}\right)$. Next we factorize the wave function in the usual radial and angular components and consider the separability provided by the first integrals $\hat{C}_{(m)}, 5.3$ with eigenvalue equations given by

$$
\Psi(r, \boldsymbol{\theta})=\Phi(r) Y(\boldsymbol{\theta}), \quad \hat{C}_{(m)} \Psi=c_{m} \Psi, \quad m=2, \ldots, N .
$$

Consequently, we obtain that $Y(\boldsymbol{\theta})$ solves completely the angular part and such hyperspherical harmonics verify

$$
\hat{C}_{(N)} Y(\boldsymbol{\theta})=\hat{\mathbf{L}}^{2} Y(\boldsymbol{\theta})=\hbar^{2} l(l+N-2) Y(\boldsymbol{\theta}), \quad l=0,1,2 \ldots
$$

where $l$ is the angular quantum number. By taking into account the angular equations (5.3), we find that the eigenvalues $c_{m}$ of the operators $\hat{C}_{(m)}$ are related to the $N-1$ quantum numbers of the angular observables as

$$
c_{k} \leftrightarrow l_{k-1}, \quad k=2, \ldots, N-1, \quad c_{N} \leftrightarrow l,
$$

that is,

$$
Y(\boldsymbol{\theta}) \equiv Y_{c_{N-1}, . ., c_{2}}^{c_{N}}\left(\theta_{1}, \theta_{2}, \ldots, \theta_{N-1}\right) \equiv Y_{l_{N-2}, . ., l_{1}}^{l}\left(\theta_{1}, \theta_{2}, \ldots, \theta_{N-1}\right) .
$$

Hence the radial Schrödinger equation provided by $\hat{\mathcal{H}}$ is

$$
\frac{1}{2\left(1+\lambda r^{2}\right)}\left(-\hbar^{2}\left(\frac{\mathrm{d}^{2}}{\mathrm{~d} r^{2}}+\frac{(N-1)}{r} \frac{\mathrm{d}}{\mathrm{d} r}-\frac{l(l+N-2)}{r^{2}}\right)+\omega^{2} r^{2}\right) \Phi(r)=E \Phi(r) .
$$

In the same way, the 1D radial Hamiltonian operators coming from the transformed LB 4.17) and PDM 4.22) quantizations are found to be

$$
\begin{aligned}
\hat{\mathcal{H}}_{\mathrm{TLB}}=- & \frac{\hbar^{2}}{2\left(1+\lambda r^{2}\right)}\left(\frac{\mathrm{d}^{2}}{\mathrm{~d} r^{2}}+\left(\frac{N-1}{r}+\frac{\lambda(N-2) r}{1+\lambda r^{2}}\right) \frac{\mathrm{d}}{\mathrm{d} r}-\frac{l(l+N-2)}{r^{2}}\right) \\
& +\frac{\omega^{2} r^{2}}{2\left(1+\lambda r^{2}\right)}-\frac{\hbar^{2} \lambda(N-2)}{8\left(1+\lambda r^{2}\right)^{3}}\left(2 N+3 \lambda r^{2}(N-2)\right), \\
\hat{\mathcal{H}}_{\mathrm{TPDM}}=- & \frac{\hbar^{2}}{2\left(1+\lambda r^{2}\right)}\left(\frac{\mathrm{d}^{2}}{\mathrm{~d} r^{2}}+\left(\frac{N-1}{r}-\frac{2 \lambda r}{1+\lambda r^{2}}\right) \frac{\mathrm{d}}{\mathrm{d} r}-\frac{l(l+N-2)}{r^{2}}\right) \\
& +\frac{\omega^{2} r^{2}}{2\left(1+\lambda r^{2}\right)}+\frac{\hbar^{2} \lambda\left(N+\lambda r^{2}(N-3)\right)}{2\left(1+\lambda r^{2}\right)^{3}} .
\end{aligned}
$$

Recall that the three radial Hamiltonians $\hat{\mathcal{H}}, \hat{\mathcal{H}}_{\text {TLB }}$ and $\hat{\mathcal{H}}_{\text {TPDM }}$, are related through the similarity transformations as

$$
\begin{aligned}
& \hat{\mathcal{H}}_{\mathrm{TLB}}=\left(1+\lambda r^{2}\right)^{(2-N) / 4} \hat{\mathcal{H}}\left(1+\lambda r^{2}\right)^{(N-2) / 4} \\
& \hat{\mathcal{H}}_{\mathrm{TPDM}}=\left(1+\lambda r^{2}\right)^{1 / 2} \hat{\mathcal{H}}\left(1+\lambda r^{2}\right)^{-1 / 2} \\
& \hat{\mathcal{H}}_{\mathrm{TPDM}}=\left(1+\lambda r^{2}\right)^{N / 4} \hat{\mathcal{H}}_{\mathrm{TLB}}\left(1+\lambda r^{2}\right)^{-N / 4} .
\end{aligned}
$$

Therefore the three corresponding radial Schrödinger equations share the same energy spectrum and have different but equivalent radial wave functions:

$$
\begin{aligned}
& \hat{\mathcal{H}} \Phi(r)=E \Phi(r), \quad \hat{\mathcal{H}}_{\mathrm{TLB}} \Phi_{\mathrm{TLB}}(r)=E \Phi_{\mathrm{TLB}}(r), \quad \hat{\mathcal{H}}_{\mathrm{TPDM}} \Phi_{\mathrm{TPDM}}(r)=E \Phi_{\mathrm{TPDM}}(r), \\
& \Phi_{\mathrm{TLB}}(r)=\left(1+\lambda r^{2}\right)^{(2-N) / 4} \Phi(r), \quad \Phi_{\mathrm{TPDM}}(r)=\left(1+\lambda r^{2}\right)^{1 / 2} \Phi(r), \\
& \Phi_{\mathrm{TPDM}}(r)=\left(1+\lambda r^{2}\right)^{N / 4} \Phi_{\mathrm{TLB}}(r) .
\end{aligned}
$$




\section{Spectrum and eigenfunctions}

In this section we shall compute, in a rigorous manner, the (continuous and discrete) spectrum and eigenfunctions of the quantum nonlinear oscillator by using the quantum Hamiltonian $\hat{\mathcal{H}}_{\text {TLB }}$ 4.22 characterized in Theorem 3. We recall that the results corresponding to the Schrödinger quantization $\hat{\mathcal{H}} 4.2$ of Theorem 2 were advanced in [6] but without explicit proofs. Recall that both quantizations share the same spectrum but they have different radial wave functions which are related through the similarity transformation (5.11).

\subsection{Continuous spectrum}

Since $\mathcal{M}^{N}$ is a complete manifold and the potential is continuous and bounded, it is standard that $\hat{\mathcal{H}}_{\mathrm{TLB}}$ is essentially self-adjoint on the space $C_{0}^{\infty}\left(\mathbb{R}^{N}\right)$ of smooth functions of compact support. It should be remarked that one cannot immediately determine the continuous spectrum of $\hat{\mathcal{H}}_{\mathrm{TLB}}$ from asymptotics of the potential: in a complete Riemannian manifold, even the spectrum of the LB operator can be extremely difficult to analyze; e.g., it can be either purely continuous (as in Euclidean space), purely discrete [45] or consist of both a continuous part and eigenvalues, possibly embedded in the continuous spectrum [46].

In fact, to compute the continuous spectrum of $\hat{\mathcal{H}}_{\mathrm{TLB}}$ it is convenient to take advantage of the spherical symmetry to decompose

$$
L^{2}\left(\mathcal{M}^{N}\right)=\bigoplus_{l \in \mathbb{N}} L^{2}\left(\mathbb{R}^{+}, \mathrm{d} \nu\right) \otimes \mathcal{Y}_{l}
$$

where $\mathrm{d} \nu(r)=r^{N-1}\left(1+\lambda r^{2}\right)^{N / 2} \mathrm{~d} r$ and $\mathcal{Y}_{l}$ is the finite-dimensional space of (generalized) spherical harmonics, defined by

$$
\mathcal{Y}_{l}:=\left\{Y \in L^{2}\left(\mathbb{S}^{N-1}\right): \Delta_{\mathbb{S}^{N-1}} Y=-l(l+N-2) Y\right\},
$$

where $\mathbb{N}$ stands for the set of nonnegative integers and $\Delta_{\mathbb{S}^{N-1}}$ denotes the Laplacian on the $(N-1) \mathrm{D}$ sphere $\mathbb{S}^{N-1}$ (or minus the angular momentum operator). This decomposition is tantamount to setting

$$
\Psi_{\mathrm{TLB}}(\mathbf{q})=\sum_{l \in \mathbb{N}} Y_{l}(\boldsymbol{\theta}) \Phi_{\mathrm{TLB}, l}(r),
$$

with $\boldsymbol{\theta}=\mathbf{q} / r \in \mathbb{S}^{N-1}, r=|\mathbf{q}|$ and $Y_{l} \in \mathcal{Y}_{l}$.

As $\hat{\mathcal{H}}_{\mathrm{TLB}}$ is spherically symmetric, the decomposition 6.1 allows us to write $\hat{\mathcal{H}}_{\mathrm{TLB}}$ as the direct sum of operators

$$
\hat{\mathcal{H}}_{\mathrm{TLB}}=\bigoplus_{l \in \mathbb{N}} \hat{H}_{\mathrm{TLB}, l} \otimes \mathrm{id}_{\mathcal{Y}_{l}}
$$

with each $\hat{H}_{\mathrm{TLB}, l}$ standing for the Friedrichs extension of the differential operator on $L^{2}\left(\mathbb{R}^{+}, \mathrm{d} \nu\right)$; namely

$$
\begin{aligned}
2 \hat{H}_{\mathrm{TLB}, l}=-\frac{\hbar^{2}}{r^{N-1}\left(1+\lambda r^{2}\right)} \frac{\mathrm{d}}{\mathrm{d} r} r^{N-1} \frac{\mathrm{d}}{\mathrm{d} r}- & \frac{\hbar^{2} \lambda(N-2) r}{\left(1+\lambda r^{2}\right)^{2}} \frac{\mathrm{d}}{\mathrm{d} r}+\frac{\hbar^{2} l(l+N-2)}{r^{2}\left(1+\lambda r^{2}\right)} \\
& +\frac{\omega^{2} r^{2}}{1+\lambda r^{2}}-\frac{\hbar^{2} \lambda(N-2)}{4\left(1+\lambda r^{2}\right)^{3}}\left(2 N+3 \lambda r^{2}(N-2)\right) .
\end{aligned}
$$


The continuous spectrum of $\hat{\mathcal{H}}_{\mathrm{TLB}}$ is most easily dealt with using this decomposition. Indeed, from 6.2 it is apparent that

$$
\operatorname{spec}\left(\hat{\mathcal{H}}_{\mathrm{TLB}}\right)=\overline{\bigcup_{l \in \mathbb{N}} \operatorname{spec}\left(\hat{H}_{\mathrm{TLB}, l}\right)}
$$

To understand the spectrum of $\hat{H}_{\mathrm{TLB}, l}$ we proceed to compute and analyze its associated quantum effective potential $\hat{\mathcal{U}}_{\text {eff }, l}$. For this purpose we apply the same change of variable $Q=Q(r)$ (3.3) used in the classical case, together with a change of the radial wave function $\Phi_{\mathrm{TLB}, l}(r) \mapsto u(Q(r))$. We require that these transformations map the Schrödinger equation $\hat{H}_{\mathrm{TLB}, l} \Phi_{\mathrm{TLB}, l}=E \Phi_{\mathrm{TLB}, l}$ into

$$
\left(-\frac{\hbar^{2}}{2} \frac{\mathrm{d}^{2}}{\mathrm{~d} Q^{2}}+\hat{\mathcal{U}}_{\mathrm{eff}, l}(Q)\right) u(Q)=E u(Q) .
$$

This is achieved by setting

$$
\Phi_{\mathrm{TLB}, l}(r)=\frac{r^{(1-N) / 2}}{\left(1+\lambda r^{2}\right)^{(N-1) / 4}} u(r)
$$

in the radial Schrödinger equation, thus yielding

$$
\hat{\mathcal{U}}_{\mathrm{eff}, l}(r)=\frac{1}{2\left(1+\lambda r^{2}\right)}\left(\frac{\hbar^{2}\left(8\left(1+\lambda r^{2}\right)-5\right)}{4 r^{2}\left(1+\lambda r^{2}\right)^{2}}+\frac{\hbar^{2}}{r^{2}}\left(l(l+N-2)+\frac{N(N-4)}{4}\right)+\omega^{2} r^{2}\right) .
$$

The behavior of $\hat{\mathcal{U}}_{\text {eff }, l}$ is rather similar to that of the classical effective potential 3.4 (see figure 4), that is, $\hat{\mathcal{U}}_{\text {eff }, l}$ is a positive function with a unique minimum, whose expression is rather cumbersome and which for the harmonic oscillator reduces to

$$
\begin{aligned}
\lambda=0: \quad & r_{\min }^{2}=\hbar \frac{\sqrt{l(l+N-2)+(N-1)(N-3) / 4}}{\omega}, \\
& \hat{\mathcal{U}}_{\mathrm{eff}, l}\left(r_{\min }\right)=\hbar \omega \sqrt{l(l+N-2)+(N-1)(N-3) / 4} .
\end{aligned}
$$

Similarly to the classical system, the values of $r_{\text {min }}$ and $\hat{\mathcal{U}}_{\text {eff }, l}\left(r_{\text {min }}\right)$ are respectively greater and smaller than those corresponding to the quantum harmonic oscillator $(6.6)$, but $\hat{\mathcal{U}}_{\text {eff }, l}$ has the same asymptotic behaviour, namely,

$$
\lim _{r \rightarrow 0} \hat{\mathcal{U}}_{\mathrm{eff}, l}(r)=+\infty, \quad \lim _{r \rightarrow \infty} \hat{\mathcal{U}}_{\mathrm{eff}, l}(r)=\frac{\omega^{2}}{2 \lambda} .
$$

We remark that there is a single exceptional particular case for $l=0$ and $N=2$ for which $\hat{\mathcal{U}}_{\text {eff }, l}$ reads

$$
\hat{\mathcal{U}}_{\mathrm{eff}, l}(r)=\frac{1}{2\left(1+\lambda r^{2}\right)}\left(\frac{-\hbar^{2}\left(1+4 \lambda^{2} r^{4}\right)}{4 r^{2}\left(1+\lambda r^{2}\right)^{2}}+\omega^{2} r^{2}\right)
$$

Thus $\lim _{r \rightarrow 0} \hat{\mathcal{U}}_{\text {eff }, l}=-\infty$ and $\lim _{r \rightarrow \infty} \hat{\mathcal{U}}_{\text {eff }, l}=\omega^{2} /(2 \lambda)$, so $\hat{\mathcal{U}}_{\text {eff }, l}$ has no minimum and can take both negative and positive values. 


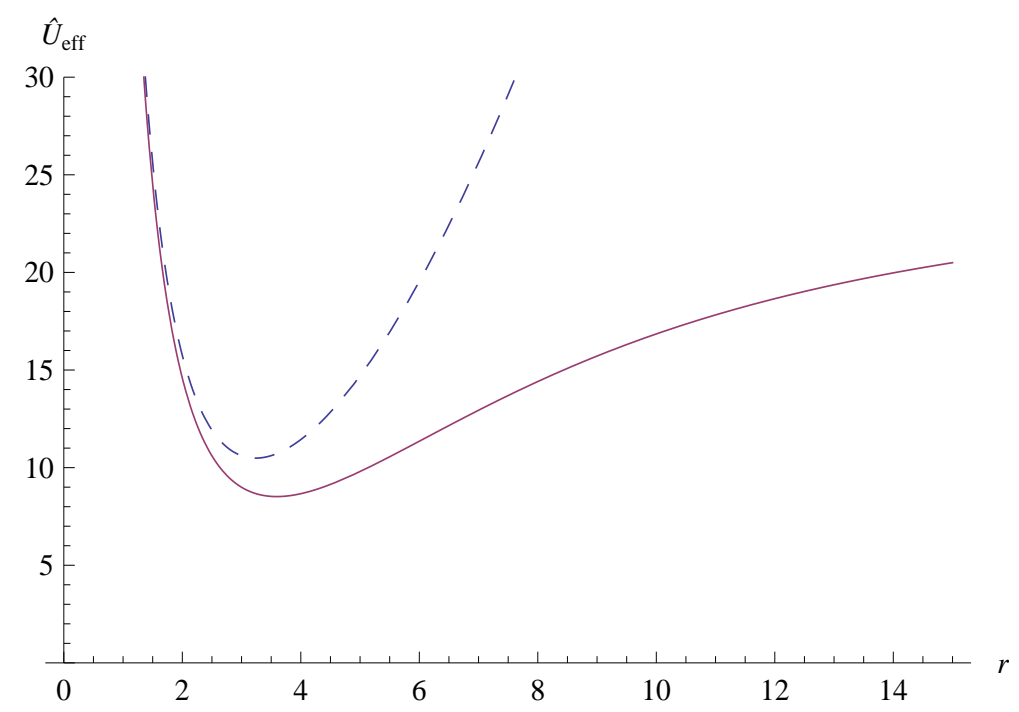

Figure 4: The quantum effective nonlinear oscillator potential (6.5) for $N=3, \lambda=0.02$, $l=10$ and $\hbar=\omega=1$. The minimum of the potential is located at $r_{\min }=3.59$ with $\hat{\mathcal{U}}_{\text {eff }, l}\left(r_{\text {min }}\right)=8.52$ and $\hat{\mathcal{U}}_{\text {eff }, l}(\infty)=25$. The dashed line corresponds to the quantum effective potential of the isotropic oscillator with $\lambda=0$ with minimum $\hat{\mathcal{U}}_{\text {eff }, l}\left(r_{\min }\right)=10.49$ at $r_{\text {min }}=$ 3.24 .

Since we have just related the nonnegative, self-adjoint second-order differential operator on the half-line $\hat{H}_{\mathrm{TLB}, l}$ to (6.3), standard results in spectral theory [47, Theorem XIII.7.66] ensure that the eigenvalues of $\hat{H}_{\mathrm{TLB}, l}$ are contained in $\left(0, E_{\infty}\right)$ and its continuous spectrum is absolutely continuous and given by $\left[E_{\infty}, \infty\right)$, where we have set

$$
E_{\infty}=\lim _{r \rightarrow \infty} \hat{\mathcal{U}}_{\mathrm{eff}, l}=\frac{\omega^{2}}{2 \lambda}
$$

Altogether, this guarantees that the continuous spectrum of $\hat{\mathcal{H}}_{\mathrm{TLB}}$ is

$$
\operatorname{spec}_{\text {cont }}\left(\hat{\mathcal{H}}_{\mathrm{TLB}}\right)=\left[\omega^{2} /(2 \lambda), \infty\right),
$$

and that there are no embedded eigenvalues.

\subsection{Discrete spectrum and eigenfunctions}

Let us now compute the eigenvalues and eigenfunctions of $\hat{\mathcal{H}}_{\mathrm{TLB}}$. To begin with, let us denote by $\psi_{n}(q)$ the $n$th eigenfunction of the $1 \mathrm{D}$ harmonic oscillator which satisfies

$$
\frac{1}{2}\left(-\hbar^{2} \frac{\mathrm{d}^{2}}{\mathrm{~d} q^{2}}+\omega^{2} q^{2}\right) \psi_{n}(q)=\hbar \omega\left(n+\frac{1}{2}\right) \psi_{n}(q)
$$

The explicit expression of $\psi_{n}$ in terms of Hermite polynomials is

$$
\psi_{n}(q)=\exp \left(-\frac{\omega}{2 \hbar} q^{2}\right) H_{n}\left(\sqrt{\frac{\omega}{\hbar}} q\right),
$$


up to a normalization constant.

Due to the relationship between the Schrödinger and LB quantizations (4.15) we have that $\Psi_{\mathrm{TLB}}(\mathbf{q})=\left(1+\lambda \mathbf{q}^{2}\right)^{(2-N) / 4} \Psi(\mathbf{q})$ and the eigenvalue equation

$$
\hat{\mathcal{H}}_{\mathrm{TLB}} \Psi_{\mathrm{TLB}}(\mathbf{q})=E \Psi_{\mathrm{TLB}}(\mathbf{q})
$$

can also be written as (see 4.2 )

$$
\left(-\hbar^{2} \Delta+\Omega^{2} \mathbf{q}^{2}\right) \Psi(\mathbf{q})=2 E \Psi(\mathbf{q})
$$

where

$$
\Omega=\sqrt{\omega^{2}-2 \lambda E}
$$

Since $\hat{\mathcal{H}}_{\text {TLB }}$ has no embedded eigenvalues (as shown in the previous subsection), one can safely assume that $\omega^{2}-2 \lambda E>0$. The condition $\Psi_{\mathrm{TLB}} \in L^{2}\left(\mathcal{M}^{N}\right)$ translates, according to 4.5 , as

$$
\int|\Psi(\mathbf{q})|^{2}\left(1+\lambda \mathbf{q}^{2}\right) \mathrm{d} \mathbf{q}<\infty
$$

in particular, $\Psi$ is square-integrable with respect to the Lebesgue measure. Therefore, by the standard theory of the harmonic oscillator, there must exist some $n \in \mathbb{N}$ such that

$$
E=\hbar \Omega\left(n+\frac{N}{2}\right) .
$$

Substituting the formula for $\Omega$, taking squares and isolating $E$, one readily finds that any eigenvalue of $\hat{\mathcal{H}}_{\mathrm{TLB}}$ must be of the form

$$
\begin{aligned}
E_{n} & =-\lambda \hbar^{2}\left(n+\frac{N}{2}\right)^{2}+\hbar\left(n+\frac{N}{2}\right) \sqrt{\hbar^{2} \lambda^{2}\left(n+\frac{N}{2}\right)^{2}+\omega^{2}} \\
& =\lambda \hbar^{2}\left(n+\frac{N}{2}\right)^{2}\left(\sqrt{1+\frac{\omega^{2}}{\hbar^{2} \lambda^{2}\left(n+\frac{N}{2}\right)^{2}}}-1\right) .
\end{aligned}
$$

Conversely, one can prove that $E_{n}$ is an eigenvalue of $\hat{\mathcal{H}}_{\mathrm{TLB}}$ for any $n \in \mathbb{N}$. This is easily seen by taking any partition $\left(n_{i}\right)_{i=1}^{N} \subset \mathbb{N}$ such that $n_{1}+\cdots+n_{N}=n$ and noticing that, by (6.8) and 6.9 ,

$$
\Psi_{\mathrm{TLB}}(\mathbf{q})=\left(1+\lambda \mathbf{q}^{2}\right)^{(2-N) / 4} \prod_{i=1}^{N} \exp \left\{-\beta^{2} q_{i}^{2} / 2\right\} H_{n_{i}}\left(\beta q_{i}\right), \quad \beta=\sqrt{\frac{\Omega}{\hbar}},
$$

is an $L^{2}\left(\mathcal{M}^{N}\right)$ solution of the equation $\hat{\mathcal{H}}_{\mathrm{TLB}} \Psi_{\mathrm{TLB}}=E_{n} \Psi_{\mathrm{TLB}}$.

Together with the result of the previous subsection, this proves the following

Theorem 5. Let $\hat{\mathcal{H}}_{\mathrm{TLB}}$ be the quantum Hamiltonian (4.22). Then:

(i) The continuous spectrum of $\hat{\mathcal{H}}_{\mathrm{TLB}}$ is given by $\left[\frac{\omega^{2}}{2 \lambda}, \infty\right)$. Moreover, there are no embedded eigenvalues and its singular spectrum is empty.

(ii) $\hat{\mathcal{H}}_{\mathrm{TLB}}$ has an infinite number of eigenvalues, all of which are contained in $\left(0, \frac{\omega^{2}}{2 \lambda}\right)$. Their only accumulation point is $\frac{\omega^{2}}{2 \lambda}$, that is, the bottom of the continuous spectrum.

(iii) All the eigenvalues of $\hat{\mathcal{H}}_{\mathrm{TLB}}$ are of the form (6.11), and $\Psi_{\mathrm{TLB}}$ is eigenfunction of $\hat{\mathcal{H}}_{\mathrm{TLB}}$ with eigenvalue $E_{n}$ if and only if it is given by a linear combination of the functions (6.12) with $n_{i} \in \mathbb{N}$ and $n_{1}+\cdots+n_{N}=n$. 


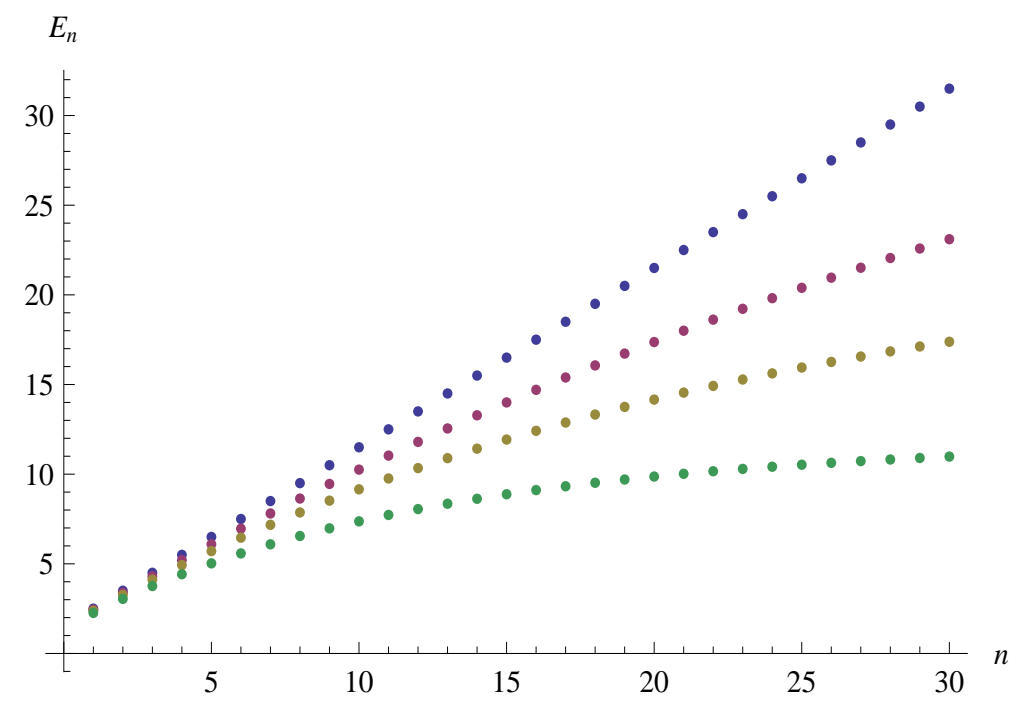

Figure 5: The discrete spectrum (6.11) for $0 \leq n \leq 25, N=3, \hbar=\omega=1$ and $\lambda=\{0,0.01,0.02,0.04\}$ starting from the upper dot line corresponding to the isotropic harmonic oscillator with $\lambda=0$; in the same order, $E_{0}=\{1.5,1.48,1.46,1.41\}$ and $E_{\infty}=\{\infty, 50,25,12.5\}$.

Therefore the bound states of this system satisfy

$$
E_{\infty}=\lim _{n \rightarrow \infty} E_{n}=\frac{\omega^{2}}{2 \lambda}, \quad \lim _{n \rightarrow \infty}\left(E_{n+1}-E_{n}\right)=0 .
$$

Such a discrete spectrum is depicted in figure 5 for several values of $\lambda$.

\section{Concluding remarks}

Summarizing, we have presented a novel exactly solvable quantum nonlinear oscillator in $N$ dimensions, that can be understood as a simultaneous "analytic" $\lambda$-deformation of both the usual isotropic oscillator potential and the underlying space on which the dynamics is defined. It turns out that if both sides of the Hamiltonian (the manifold and the potential) are appropriately modified, the curved quantum system preserves all the superintegrability properties of the Euclidean one, and its full solution can be explicitly obtained by making use of the curved analogues of Fradkin operators. It is worth stressing that such an explicit solution could be of interest from the physical viewpoint, since a parabolic effective-mass function has been proposed in [48, 49] in order to describe realistic quantum wells formed by semiconductor heterostructures.

On the other hand, the quest for the preservation of superintegrability under quantization seems to be also a valuable guideline in order to clarify the properties of different possible quantization recipes on generic Riemannian manifolds. In this sense, we think that the connection here presented between the conformal Laplacian approach and the MS property is worth to be investigated through, for instance, the study of the quantization of other MS systems on spaces of nonconstant curvature that have been recently characterized in the context of the generalized Beltrami theorem [33]. 
Finally, we recall that the real parameter $\lambda=1 / \kappa$ was restricted in 3 to take a positive value. However, the MS of the classical Hamiltonian stated in Theorem 1 does hold for negative $\lambda$ as well. Nevertheless, the underlying space and the oscillator potential change dramatically when $\lambda<0$ (see [35]), and the corresponding quantum problem is currently under investigation by making use of the techniques here presented.

\section{Acknowledgments}

This work was partially supported by the Spanish MICINN under grants MTM2010-18556 and FIS2008-00209, by the Junta de Castilla y León (project GR224), by the Banco SantanderUCM (grant GR58/08-910556) and by the Italian-Spanish INFN-MICINN (project ACI20091083).

\section{References}

[1] A. Ballesteros, A. Enciso, F.J. Herranz, O. Ragnisco, Phys. Lett. B 652 (2007) 376.

[2] A. Ballesteros, A. Enciso, F.J. Herranz, O. Ragnisco, Ann. Phys. 324 (2009) 1219.

[3] A. Ballesteros, A. Enciso, F.J. Herranz, O. Ragnisco, Physica D 237 (2008) 505.

[4] D.M. Fradkin, Amer. J. Phys. 33 (1965) 207.

[5] T. Iwai, Y. Uwano, N. Katayama, J. Math. Phys. 37 (1996) 608.

[6] A. Ballesteros, A. Enciso, F.J. Herranz, O. Ragnisco, D. Riglioni, Phys. Lett. A (2011) to appear, preprint arXiv:1007.1335.

[7] R.M. Wald, General Relativity, The University of Chicago Press, Chicago, 1984.

[8] N.P. Landsman, Mathematical Topics Between Classical and Quantum Mechanics, Springer, New York (1998).

[9] Z.J. Liu, M. Qian, Trans. Amer. Math. Soc. 331 (1992) 321.

[10] C. Baer, M. Dahl, Geom. Funct. Anal. 13 (2003) 483.

[11] S.M. Paneitz, SIGMA 4 (2008) 036.

[12] G. Bastard, Wave mechanics applied to semiconductor heterostructures, Les Éditions de Physique, Paris (1988).

[13] P. Harrison, Quantum wells, wires and dots, Wiley, New York (2009).

[14] O. von Roos, Phys. Rev. B 27 (1983) 7547.

[15] J.M. Lévy-Leblond, Phys. Rev. A 52 (1995) 1845.

[16] C. Quesne, V.M. Tkachuk, J. Phys. A: Math. Gen. 37 (2004) 4267.

[17] C. Quesne, Ann. Phys. 321 (2006) 1221. 
[18] G. Koenigs, in: Leçons sur la théorie générale des surfaces vol. 4, ed. G. Darboux, Chelsea, New York, 1972, p. 368.

[19] E.G. Kalnins, J.M. Kress, W. Jr. Miller, P. Winternitz, J. Math. Phys. 44 (2003) 5811.

[20] T. Iwai, N. Katayama, J. Math. Phys. 36 (1995) 1790.

[21] D. Zwanziger, Phys. Rev. 176 (1968) 1480.

[22] H.V. McInstosh, A. Cisneros, J. Math. Phys. 11 (1970) 896.

[23] N.S. Manton, Phys. Lett. B 110 (1982) 54.

[24] M.F. Atiyah, N.J. Hitchin, Phys. Lett. A 107 (1985) 21.

[25] G.W. Gibbons, N.S. Manton, Nucl. Phys. B 274 (1986) 183.

[26] L.G. Fehér, P.A. Horváthy, Phys. Lett. B 183 (1987) 182.

[27] G.W. Gibbons, P.J. Ruback, Comm. Math. Phys. 115 (1988) 267.

[28] D. Bini, C. Cherubini, R.T. Jantzen, Class. Quantum Grav. 19 (2002) 5481.

[29] D. Bini, C. Cherubini, R.T. Jantzen, B. Mashhoon, Class. Quantum Grav. 20 (2003) 457.

[30] V. Perlick, Class. Quantum Grav. 9 (1992) 1009.

[31] A. Ballesteros, A. Enciso, F.J. Herranz, O. Ragnisco, Class. Quantum Grav. 25 (2008) 165005.

[32] J. Bertrand, C. R. Acad. Sci. Paris 77 (1873) 849.

[33] A. Ballesteros, A. Enciso, F.J. Herranz, O. Ragnisco, Commun. Math. Phys. 290 (2009) 1033.

[34] J.P. Ngome, J. Math. Phys. 50 (2009) 122901.

[35] A. Ballesteros, A. Enciso, F.J. Herranz, O. Ragnisco, D. Riglioni, (2010) preprint arXiv:1010.3358

[36] J. Hietarinta, B. Grammaticos, B. Dorizzi, A. Ramani, Phys. Rev. Lett. 53 (1984) 1707.

[37] E.G. Kalnins, J.M. Kress, W. Jr. Miller, J. Math. Phys. 46 (2005) 053510.

[38] E.G. Kalnins, J.M. Kress, W. Jr. Miller, J. Math. Phys. 47 (2006) 043514

[39] E.G. Kalnins, W. Jr. Miller, S. Post, J. Phys. A: Math. Theor. 43 (2010) 035202.

[40] A. Ballesteros, O. Ragnisco, J. Phys. A: Math. Gen. 31 (1998) 3791.

[41] O. Ragnisco, A. Ballesteros, F.J. Herranz, F. Musso, SIGMA 3 (2007) 026.

[42] A. Ballesteros, A. Blasco, F.J. Herranz, F. Musso, O. Ragnisco, J. Phys.: Conf. Series 175 (2009) 012004 .

[43] R.S. Strichartz, J. Funct. Anal. 52 (1983) 48.

[44] M.A. Shifman, A.V. Turbiner, Commun. Math. Phys. 126 (1989) 347.

[45] H. Donnelly, P. Li, Duke Math. J. 46 (1979) 497.

[46] H. Donnelly, Michigan Math. J. 28 (1981) 53. 
[47] N. Dunford, J.T. Schwartz, Linear Operators II, Wiley, New York, 1988.

[48] R. Koc, M. Koca, G. Sahinoglu, Eur. Phys. J. B 48 (2005) 583.

[49] A.G.M. Schmidt, Phys. Lett. A 353 (2006) 459. 